\title{
The oceanic nature of the African slab subducted under Peloponnesus: thin-layer resolution from multiscale analysis of teleseismic $P$-to- $S$ converted waves
}

\author{
A. Gesret, ${ }^{1,2}$ M. Laigle, ${ }^{1}$ J. Diaz, ${ }^{3}$ M. Sachpazi ${ }^{4}$ and A. Hirn ${ }^{1}$ \\ ${ }^{1}$ Sismologie, Institut de Physique du Globe de Paris, UMR 7514 CNRS, 4 place Jussieu, 75 Paris, France. E-mail: alexandrine.gesret@mines-paristech.fr \\ ${ }^{2}$ Mines ParisTech, Centre de Géosciences, 77 Fontainebleau, France \\ ${ }^{3}$ Consejo Superior de Investigaciones Cientificas, Instituto Jaume Almera, c/Sole i Sabaris, Barcelona, Spain \\ ${ }^{4}$ National Observatory of Athens, Geodynamical Institute Lofos Nymfon, Athens, Greece
}

Accepted 2010 July 15 . Received 2010 July 2; in original form 2009 September 7

\begin{abstract}
SUMMAR Y
In the Hellenic subduction zone, the lithospheric slab may comprise continental and oceanic units juxtaposed downdip and along strike. For stations along eastern Peloponnesus, teleseismic $P$-wave receiver-function (RF) processing in the standard frequency band produces an image of a low-velocity layer (LVL) at the top of the slab apparently twice thicker than for an oceanic crust. To assess if this could come from a lack of resolution of the standard processing, we develop a multiscale approach with the RFs based on the wavelet-response of the medium, akin to the wavelet-transform of the velocity-depth function. The synthetic response in conversion is obtained for a multiscale singularity formed by two opposite velocity-steps at the boundaries of a crust embedded in mantle material. This indicates that only wavelet periods shorter than about $0.8 \mathrm{~s}$ will allow to identify clearly a $7 \mathrm{~km}$ thin oceanic crust. Going to longer periods leads to underestimate or overestimate the time-thickness of the LVL, due to interference phenomena. The analysis of the response in conversion from full waveform synthetic seismograms in a dipping slab model validates a multiresolution approach to real observations. With earthquakes of broad-enough spectrum towards high frequencies, yielding energy to provide wavelet periods significantly shorter than $1 \mathrm{~s}$, the $P$-to- $S$ conversions obtained allow us to resolve for the first time a standard oceanic crust at the slab top beneath the eastern coast of Peloponnesus. This documents the subduction of a purely oceanic slab of most reduced buoyancy since 4-5 Myr under the rapidly southwestward extending upper plate continental material.
\end{abstract}

Key words: Wavelet transform; Body waves; Subduction zone processes; Dynamics of lithosphere and mantle; Africa; Europe.

\section{INTRODUCTION}

Under Greece, oceanic and continental lithospheric segments have been subducted in succession, or lateral juxtaposition along the Hellenic subduction zone (Fig. 1), according to geological evidence (e.g. van Hinsbergen et al. 2005). In accordance to their nature, the subducted plate segments differ by their crustal thickness, superficial structure and buoyancy as expressed in the depth under water of their top before entering subduction (e.g. Royden 1993). These differences induce corresponding variations in the geometry and the nature of the interaction between the subducting material and the medium above the slab. Slab buoyancy variations likely control transients like trench retreat or slab rollback when oceanic lithosphere of strongest negative buoyancy enters subduction. Such variations may induce different behaviours in space and time at the subduction zone that have major significance for the geodynamic processes (Royden \& Husson 2006) and also for the seismic coupling of the seismogenic part of the interplate (Laigle et al. 2002). This subduction zone is indeed prone to $M 8$ earthquakes, such as occurred for example at the turn of the 19th-20th century (e.g. Wyss \& Baer 1981), or even greater ones as the 365 AD Gortyn earthquake (Laigle et al. 2004).

In the specific case of the Aegean domain, past or presently active deformation of the upper plate is rather complex in time and space and may not be fully understood if the only forces considered are those applied at the plate boundaries seen at the surface. Indeed the slab present underneath may also control the evolution, especially if its structure and behaviour are heterogeneous.

The characteristics of the crust at the top of the slab are diagnostic of the nature of the slab, with the oceanic case of strongest negative buoyancy corresponding to the smallest thickness, of only $7 \mathrm{~km}$, of low density crust (White et al. 1992). However, the depth resolution 

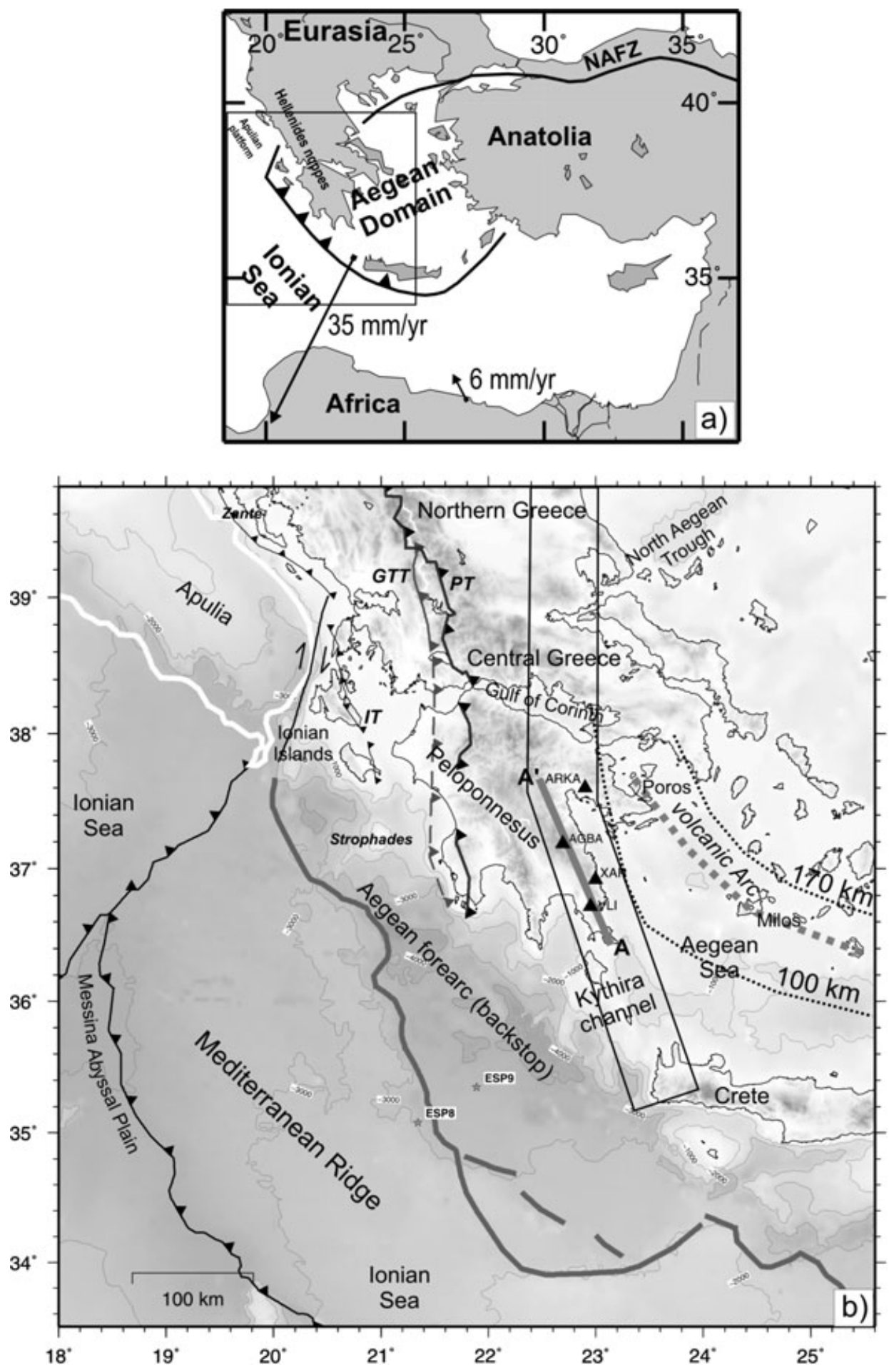

Figure 1. (a) Sketch of the Hellenic subduction zone in the wider context, with the vectors of instantaneous velocity of the SW Aegean domain and the African continent derived from GPS (after Kahle et al. 2000). (b) Map of the Hellenic subduction region with black triangles indicating the particular position of seismometers for which data are shown (Figs 9 and 10) and the $150 \mathrm{~km}$ segment AA' of the image section of 300 receiver-functions over eight stations in Fig. 2, within the swath of stations deployed in the 'THALES WAS RIGHT' project. Main tectonic elements related to the convergence are sketched: from SW to NE. Full barbed line: External limit, deformation front at the frontal thrust of the Mediterranean ridge accretionary wedge over the deep oceanic basin of the Messina Abyssal plain in the Ionian Sea, that is the northern margin of the African Plate. Grey bold line offshore: limit (generally a local backthrust in its shallow part, e.g. Chamot-Rooke et al. 2005) between the accretionary wedge of the Mediterranean Ridge and the backstop that is the Aegean forearc. CF indicated west of the Ionian Islands in northward continuation of the two previous lines is the presently active Cephalonia fault, strike-slip limit at surface with the Apulian continuation of Africa and linking northward with compressional structures where collision has followed the end of subduction in the Dinarides. Barbed lines further inland mark frontal thrusts of Hellenic nappe, labelled IT for the Ionian thrust of the Ionian Zone on the Pre-Apulian, active from 13 Ma, and GTT for the Gavrovo-Tripolis and PT for the Pindos thrust active earlier. Dotted lines labelled 100 and $170 \mathrm{~km}$ are proposed isobath for the Wadati-Benioff earthquakes (Papazachos et al. 2000) that encompass the volcanic Arc marked by line with big grey squares. 
of the top of this slab obtained by regional or global traveltime tomography is no better than tens of kilometres (e.g. Spakman 1986; Spakman et al. 1993). On the other hand, active source seismics with combined multichannel seismic imaging and wide-angle refraction and reflection modelling have the required resolution, but their penetration seems limited to depths shallower than $25 \mathrm{~km}$ in the Hellenic subduction zone. The precise location and nature of the slab top cannot either be well resolved by the intermediate depth earthquakes of the Wadati-Benioff zone. Their hypocentres are not constrained to better than tens of kilometres, since their magnitudes are generally too low for a good worldwide recording.

In order to evaluate the specific behaviour of this particular subduction zone, we need a specific seismic approach that resolves the nature of the slab segments at depth, which may vary from continental to oceanic.

Incoming teleseismic body waves, recorded by seismometers located above the target at the Earth's surface, undergo forward scattering by mode conversion. The first arriving $P$ wave is thus followed by the $S$ converted from it at singularities in the velocity-depth function, of which the simplest is the step function at interfaces between layers. The receiver function (RF) method applied on the recordings allows us to retrieve these mode-converted waves. For the case of the crust on top of a slab that is already subducted deeper than the upper plate Moho, and thus forms a low-velocity layer (LVL) between the mantle material of the upper plate and that of the slab, two opposite polarity $S$ arrivals converted from $P$ are expected in the first seconds of the $P$ coda. Their time interval or spacing is hence a measure of the thickness of the slab crust, with their amplitudes being a function of the contrast at conversion boundaries, mainly of the $S$ velocity. Recently, RF or scattered wave analyses across subduction zones and related new imaging techniques could be used to resolve the crust at the top of the slab and the domain of interaction with the overlying mantle (Ferris et al. 2003; Rondenay et al. 2008). However, these studies reported apparently much thicker LVL at the slab top than expected for an oceanic crust, and discussed the far-reaching implications of such results.

We present a new approach to resolve the fine nature of heterogeneities encountered at depth and to image the LVL located at the top of the subducting slab. We adapted a multiresolution approach by wavelet-analysis to the case of converted waves and applied it to the conventional RF processing approach, which uses forward-scattered $S$ waves converted from teleseismic $P$ waves. Single-station analysis is necessary as a first step to avoid blurring the accuracy and resolution. The multiresolution analysis will demonstrate that interactions between the signal wavelength and the LVL thickness may prevent an accurate determination of the nature of the heterogeneity. This analysis thus reveals that for such a thin inclusion, special attention is required to check that forward-scattered teleseismic body waves can provide us with the necessary signal wavelength.

We also present the first results of the multiresolution approach as applied to examples of the data set collected with the objective of better constraining the structural control of the large subductionearthquakes segmentation. This data set and study is part of an ongoing project along the Western Hellenic Arc that includes support in the frame of the 'THALES WAS RIGHT' project of the European Commission FP6 NEST-INSIGHT programme. The receivers have been installed in a swath from western Crete to central Greece, in particular along the eastern coast of Peloponnesus. In this region, the question of the nature of the slab top LVL with respect to the presence of an oceanic crust can be specifically addressed and results used to track its spatial variation, which is indeed crucial to discuss the models of the geodynamic evolution of the region.

The strike-line swath profile in our project contributes to the general coverage of the region in connection with the NSF MEDUSA project in Greece. Their dip-line runs from the middle of the western coast of Peloponnesus, onshore at the latitude of the Strophades islands (Fig. 1) through the isthmus east of the Gulf of Corinth. From their multimode imaging comprising the $P$-to- $S$ converted waves and in addition the surface-backscattered and converted waves, Suckale et al. (2009) suggest at the top of the slab an LVL with relatively constant thickness of $15-22 \mathrm{~km}$ and dip of $21^{\circ}$ towards east. They report along-strike slab variations close to their dip-line, from observed variations of results with backazimuths.

The first observations we report here are obtained south of such a dip-line from Strophades to Corinth, up to $100 \mathrm{~km}$ along the strike of the structural trend at the surface along eastern Peloponnesus. This is the subducted lithospheric panel downdip the largest well documented earthquake from the subduction zone for which the location and extent of the rupture zone have been inferred, the M 7.9, 1903 so-called Kithira earthquake (e.g. Wyss \& Baer 1981).

\section{GEODYNAMIC, STRUCTURAL AND SEISMOLOGICAL CONTEXT}

\subsection{Upper plate tectonics and structure}

Between the two large plates, Europe and Africa (Fig. 1), that converge slowly at less than $10 \mathrm{~mm} \mathrm{yr}^{-1}$ and the Anatolia subplate that rotates in-between (e.g. Le Pichon et al. 1995), the Aegean domain does not appear as a regular plate, since it does not meet the criterion of a rigid body limited by plate-boundary faults. The South-Aegean domain, which has undergone extension in rather recent times, does not deform internally any more at present, while it travels southward four times faster than the large plates converge. The present internal deformation shown by GPS is in a rather broad region along the northeastern and northwestern transitions of this Aegean domain, respectively to Anatolia on one side, and to Europe (i.e. Northern Greece) on the other side (e.g. McClusky et al. 2000; Nyst \& Thatcher 2004; Hollenstein et al. 2008). These rather broad surroundings of the Aegean domain are furthermore the places of highest seismic activity of Europe and the Mediterranean domain.

This Aegean upper plate, and its ill-defined northwestern boundary also appear characterized by strong variations in crustal thickness that may be related to processes deeper in the lithosphere and asthenosphere. From the Pn traveltime anomalies of a major local earthquake recorded by a dense array, Sachpazi et al. (2007) document a line striking SW-NE from the western Gulf of Corinth towards the North Aegean Trough as being a contact between thinned crust in the southeastern area and thicker crust in the northwestern one.

These limits thus happen to coincide with structures of LateMiocene to Early Pliocene deformation, such as the Parnon trenchparallel extensional detachment running SSE through Eastern Peloponnesus, as they have been derived from surface geology by Papanikolaou \& Royden (2007). The independently reached findings of the limits of the high in the Moho-depth map of Sachpazi et al. (2007) and of the tectonic identification of a CHSZ, Central Hellenic Shear zone by Papanikolaou \& Royden (2007) clearly concur to defining a region of thinned crust with two limits. One is running from the middle of the Gulf of Corinth towards ENE as the 
northern limit of the CHSZ, and the other towards SSE to southeastern Peloponnesus.

\subsection{Top of the subducting plate at shallow depth}

The depth variation of the top of the African slab under the region, and thus the values of dip and azimuth of the subducted slab are not tightly constrained from previous studies. There is a variation along the arc of both the strike of the sea-bottom trace of the frontal thrust at the tip of the accretionary wedge (Fig. 1b, barbed line) and of the strike of its inner boundary with the backstop (Fig. 1b, full grey line). In addition, the difference among these strikes is variable along the arc in relation with the variation of slip partitioning and deformation in the wedge (e.g. Le Pichon et al. 1995). However, for the particular segment offshore SW Peloponnesus, their strikes are the same, about $\mathrm{N} 120^{\circ} \mathrm{E}$. At the greater depth of $100 \mathrm{~km}$, the slab depth contour (Fig. 1b, dotted lines) inferred from seismicity (Papazachos et al. 2000) is almost N-S along eastern Peloponnesus, whereas the volcanic arc, supposedly above an isobath of the deeper slab, is again striking $\mathrm{N} 120^{\circ} \mathrm{E}$ between Poros and Milos volcano islands.

The direction and magnitude of slab dip may thus not be constant and whether they may vary smoothly or abruptly, such as by juxtaposing segments of slab along strike, is not documented. In case of such a geometrical segmentation of the slab, this may affect that of the interplate thrust. Indeed, a geometrical segmentation may result in variations of the seismic coupling along the seismogenic part of this interplate boundary at the top of the slab and therefore be of relevance to the question of major earthquakes.

The interplate boundary has been evidenced and imaged by a few dedicated marine seismic projects that pushed the active source marine seismics exploration methods from surface to largest depth, with the aim to learn more on this seismogenic part.

In the Ionian Islands region, marine multichannel reflection seismic (MCS) profiling between Cephalonia and Zante (Hirn et al. 1996; Clément et al. 2000) imaged a reflector, dipping landward from around 13 to $15 \mathrm{~km}$ depth. Since this level happened to correspond to a limit between microearthquakes of different mechanisms (Sachpazi et al. 2000) and to the location of the major instrumentally located earthquakes, it was suggested to mark the locked interplate boundary that could be the source of the earthquakes of magnitude above 7 that occur once or twice a century (Laigle et al. 2002). Offshore western Crete, flat thrust earthquakes relocated at $40 \mathrm{~km}$ depth (Taymaz et al. 1990) may indicate the depth to the top of the subducting plate there. None of those data has provided a clear clue on the nature of the medium beneath the interplate boundary and on the upper part of the lower plate before subduction, in this zone close to the termination of the subduction against the Cephalonia fault.

Beginning on the lower plate before it enters subduction, a more complete section across the subduction has been obtained since then, in the southwestern offshore of southern Peloponnesus. In the long and narrow Messina abyssal plain where the top of the Ionian oceanic lithosphere is at $4000 \mathrm{~m}$ water depth, several MCS profiles across the deformation front reveal a 3-4 km thick sedimentary cover above the oceanic basement top (Reston et al. 2002; Polonia et al. 2002). There, the offscraping process of the sediments of the Ionian subducting lithosphere and their accretion against the Aegean backstop results in the huge deforming accretionary wedge of the Mediterranean Ridge above the interplate décollement. The seismic images show that at the deformation front, the interplate be- neath which the subducting lithosphere is underthrusting, generally lies at the base of the Messinian evaporites. There is evidence that it may even step further down into deeper levels of the sedimentary cover at short distance of the deformation front. At the landward deformation front of the accretionary wedge against the backstop 200 $\mathrm{km}$ further East, reinterpretation of ESP profiles (ESP 9 in Fig. 1) and $\mathrm{OBH}$ wide-angle and refraction modelling have identified the interplate boundary at $14 \mathrm{~km}$ depth. The top of the subducting plate is interpreted as comprising sediments of only 1-2 km thickness, remaining above the igneous oceanic crust (de Voogd et al. 1992; Truffert et al. 1993; Jones et al. 2002). This suggests that the slab subduction may not entrain an important thickness of sediments on top the igneous oceanic crust. In this case, there is no reason for the LVL between the slab mantle and the overlying mantle to be significantly thicker than a standard oceanic crust.

\subsection{Nature of the subducting plate}

In the northwestern Hellenides of northern Greece (Fig. 1) and the Dinarides further north, it is widely accepted that the subduction has halted and turned into collision, since there is no further oceanic lithosphere to consume.

Based on seismic tomographic images (e.g. Spakman et al. 1993), Wortel \& Spakman (2000) have suggested that there, the slab would have been broken off from above, thus causing a segmentation at large scale of the subduction zone. In the northernmost, now continental-collision part, the deeper part of the slab, already detached from above, would have remained attached laterally to its southern prolongation (Wortel \& Spakman 2000). The lateral overweight thus exerted at depth on that part of the slab which is still attached and in continuity with the Ionian basin lithosphere from the surface, might influence the trench retreat, the seismic coupling and the motion and deformation of the upper plate, and modulate them from the Dinarides to Crete (Laigle et al. 2002, 2004).

Global and regional seismic tomographic images do not distinguish internal heterogeneity within the large volume of subducted lithosphere under the Aegean. However, there is geological evidence in the recent evolution for successive consumption of three oceanic basins and docking of two continental blocks (e.g. van Hinsbergen et al. 2005) that are now in the shallow part of the tomographic anomaly that reaches $1400 \mathrm{~km}$ deep. Such subduction of a succession of different lithospheric segments has been inferred from the recent orogenic evolution of the Hellenides. This has been described as a foreland-propagating fold and thrust belt (e.g. Underhill 1989). Its front jumped in middle Miocene $13 \mathrm{Ma}$ from a position at the Pindos thrust over the Gavrovo-Tripolis zone to a position west of the Ionian zone (Fig. 1). There, the corresponding Ionian Thrust over the Paxos or Pre-Apulian zone outcrops on the Ionian Islands where at present it is not anymore the active subduction boundary. In the Ionian Islands segment, the frontal thrust is considered to have jumped 4-5 Ma ago further to the west of the southern island of Zante and the Strophades islets. In this region, the southern part of the pre-Apulian continental domain now actively overthrusts the deep Ionian basin lithosphere with its oceanic crust.

The pre-Apulian continental domain does not outcrop at present further south than the Ionian Islands along the Hellenic Arc, and there is no evidence that it would have extended further south. This is of particular relevance for our study of the subducted slab, since it suggests that the underthrusting and subduction of the lithosphere of the deep Ionian basin sea could indeed have occurred earlier 
under southern Peloponnesus and the Kythira channel than it did in the Ionian Islands.

\section{OBJECTIVE OF THE STUDY, TARGET AND DESIGN OF THE SURVEY}

\subsection{Objective of the RF slab imaging study}

A major support to a model in which the present regime corresponds to fastest trench retreat and slab rollback occurring in the southern part of the Western Hellenic subduction, would be that the corresponding slab has the largest negative buoyancy. This is the case for a slab segment of purely oceanic lithosphere.

Such fast trench retreat due to an oceanic slab could have been occurring since at least $4 \mathrm{Ma}$ ago, in relation with the migration or jump of the subduction front. The latter migrated from its position as the Ionian Thrust of the Ionian Zone over the pre-Apulian domain that was not of oceanic lithosphere and is now outcropping through the Ionian Islands, to a position of this subduction thrust that would be now in the marine domain to the west of these islands. If the present rate of convergence of $30-40 \mathrm{~mm} \mathrm{yr}^{-1}$ across the subduction boundary between Africa and the south Aegean domain (not Europe as a whole) applied since $4 \mathrm{Ma}$, the slab crust subducted then would be now at a $120-160 \mathrm{~km}$ distance northeast of the present subduction backstop tip offshore southwestern Peloponnesus. This corresponds to the eastern coast of Peloponnesus. We hence deployed there several stations of our seismic array in order to investigate this possible oceanic nature of the slab by testing specifically its crust. We are also carrying on observations both towards north and south in order to detect along-strike variations.

Oceanic lithosphere is characterized by the thinnest crust at its top. Whether, when subducted to large depths, a resulting LVL as thin as its $7 \mathrm{~km}$ can be identified is a major question in seismic imaging. Indeed, a much larger thickness of low-velocity zones at the slab top region appears in images obtained recently from the supposedly most resolving approach with body waves that are forward converted or scattered from incoming teleseismic $P$ waves. This is the case in Alaska (Ferris et al. 2003, Rondenay et al. 2008), in the Cascadia subduction of Oregon (Bostock et al. 2002) and in Vancouver (Nicholson et al. 2005). We will similarly use body waves from distant earthquakes that are mode-converted at the top of the slab, but will present here a new method to reach in a quantifiable way the high-resolution required.

\subsection{Design of the survey and data set}

Current teleseismic sources at adequate distances of the study region are located in Kuriles, Kamchatka, Japan, western Pacific and Indonesian subduction zones and thus confined to northeastern backazimuths, ranging from $\mathrm{N} 10^{\circ} \mathrm{E}$ to $\mathrm{N} 100^{\circ} \mathrm{E}$. For a slab dipping grossly to Northeast as expected here, a single line of receivers in this direction is then aligned along dip and with the earthquakes, and most adequate for the purpose of investigating the slab attitude and variation with depth. Instead, in order to search for variations in the slab attitude along the subduction zone, such as tears and segmentation of the slab, observations are needed along a NW-SE strike-line.

We concentrate here on the fine analysis of the $S$ converted forward-scattered from $P$ at the slab top that arrive shortly after $P$. This phase is free from the interference with the response of the deeper structure. This is not the case for the much later arriving so-called multiples from the slab top that could be used also, since in addition they have interacted with the heterogeneity near the surface from which they are backscattered. For the slab depths expected here, on the order of $60 \mathrm{~km}$, the response in $P$-to- $S$ conversion of the slab crust will also be earlier than, and thus non-interfering with the multiples of the upper plate crust conversions that can be so strong that they have been used to constrain further parameters such as $V_{P} / V_{S}$ of that crust (e.g. Lombardi et al. 2008).

A profile of $500 \mathrm{~km}$ has been deployed from Crete to Central Greece (Fig. 1), with $\mathrm{N} 150^{\circ} \mathrm{E}$ direction South of the Gulf of Corinth, and veering direction to the North further north. This could be done within the EU 'THALES WAS RIGHT' project beginning in mid 2006, with different pools of seismological stations comprising Hathor, Reftek, Marslite and Titan dataloggers equipped with 5, 20, 40 or $120 \mathrm{~s}$ Lennartz, Güralp and Streckeisen sensors. The instrument number has fluctuated over time between 20 and 40 . We have redeployed them several times in different sites in order to improve spatial coverage or to better constrain some localised complex regions identified in the preliminary results. They are still in operation at the time of writing, in order to complete the survey.

The Z-N-E three-component waveforms of recorded teleseismic events are projected into the local $P-S v-S h(\mathrm{~L}-\mathrm{Q}-\mathrm{T})$ ray-based coordinate system thus isolating the $P$ waves on the $\mathrm{L}$ component along the impinging first arrival, and the $S$ waves converted from $P$ at horizontal interfaces on the $\mathrm{Q}$, or $\mathrm{Sv}$ component. (To obtain the RF, the $S v$ component is then deconvolved by the $P$ component signal.) We use the methods implemented in Seismic Handler (Stammler 1993; Kind et al. 1995) to obtain from observed seismograms the wavelet of the $P$ and by deconvolution, the wavelet of the $S v$ converted from $P$, that is the receiver function, RF and also to generate synthetics.

\section{ANALYSIS OF REAL OBSERVATIONS AND WAVELET ANALYSIS APPROACH}

\subsection{Evidence for the slab top from standard RF processing}

Interpretations of RF and scattered waves studies are often based on images resulting from migration of RF amplitude into a time- or depth-section along a profile. For about $300 \mathrm{RF}$ data of 8 stations along a $150 \mathrm{~km}$ long line (Fig. 1), we applied such a migration with the same processing as Yuan et al. (2000) who reported a 10-kmthick low-velocity zone at the top of the Pacific oceanic slab under the Andes. Here in Fig. 2, we have chosen a symmetrical colour scale for positive and negative variations of velocity with depth, as Li et al. (2000) for the Japan subduction, in order to clearly identify the velocity contrast with increasing depth at the slab crust top as well as at its base, by their respective blue negative, and red positive.

On this densely documented profile of Fig. 2, apart from the positive polarity converted waves at the Agean Moho at around $3 \mathrm{~s}$, the clearest feature is the positive of the conversion at the lithospheric slab Moho arriving between 6.5 and $8.5 \mathrm{~s}$. About $2 \mathrm{~s}$ earlier, an almost equally strong negative polarity can thus be associated with the top of the slab crust under the overlying upper plate mantle. With these two opposite polarity signals we obtain quantitative data for a discussion of the slab crust and thus its nature.

The migrated image of Fig. 2 is obtained from data filtered with a $0.5 \mathrm{~Hz}$ high-frequency cut-off. Commonly, the high-frequency cutoff is even $0.3 \mathrm{~Hz}$ hence neglecting signal periods shorter than $3 \mathrm{~s}$ (e.g. Nicholson et al. 2005; Rondenay et al. 2008). The latter filter is applied in the example of the RF in the figure, of a $M_{\mathrm{w}}=6.6$ 

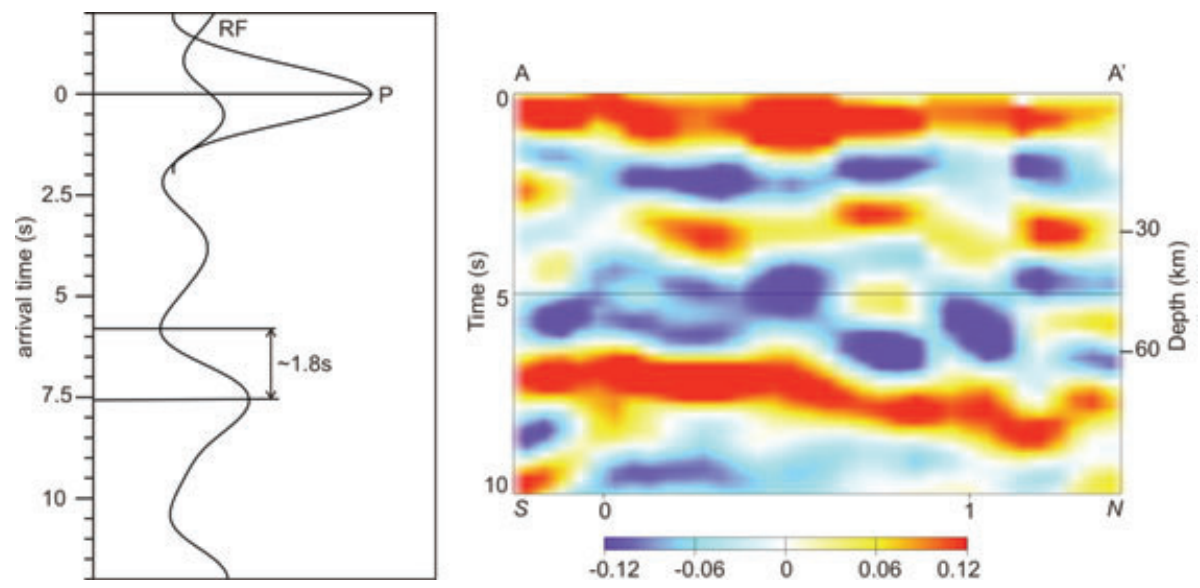

Figure 2. On the left is the receiver-function obtained by deconvolution of the $S v$ wave on the $\mathrm{Q}$ by the $P$ wave on the $\mathrm{L}$ component. Trace noted RF is indeed that of $S$ waves converted from the incoming teleseismic $P$, of which the deconvolved zero-phase wavelet is shown as trace noted $P$. Traces for a $M_{\mathrm{w}} \sim 6.6$ Kuril earthquake (2006 September 30, backazimuth $32^{\circ}$, distance $86^{\circ}$ ) recorded at the VLI (Fig. 1) station obtained after application to the seismograms of a commonly used filter of passband 3-20 s. On the right, migrated image of $300 \mathrm{RF}$ of eight stations from south to north along the $150 \mathrm{~km}$ line AA' shown in Fig. 1. Time-section, the corresponding depth is also indicated on the right-hand scale (crust at $V_{P}=6.3 \mathrm{~km} \mathrm{~s}^{-1}$ from 0 to $30 \mathrm{~km}$ depth, mantle at $V_{P}=8$ $\mathrm{km} \mathrm{s}^{-1}$ ). VLI is at the southern third of the line.

Kuril earthquake (2006 September 30 17h50m23s) recorded at the VLI permanent seismological station of the National Observatory of Athens (see Fig. 1) equipped with a seismometer with flat velocity response up to $20 \mathrm{~s}$ period (this has been replaced since by a standard $60 \mathrm{~s}$ period broad-band sensor Güralp 3ESPC/60 used for instance in Fig. 10, that confirms the analyses made in this study). The resulting RF shows a clear signature of the top of the Hellenic slab arriving around $6 \mathrm{~s}$ later than the first arrival $P$, that is a conversion from about $50 \mathrm{~km}$ depth (Fig. 2). It marks as a negative, a trough, followed by a positive, a peak. This is typical of a LVL, as expected for the crust at the top of the lithospheric slab underneath the Aegean mantle. The RF amplitude with respect to $P$ wavelet, that is the amplitude of the $S v$ converted from $P$ is larger than for the mere velocity contrast of upper plate crust on slab mantle material. This is expected because of the updip incidence on the inclined slab from the northeastern backazimuths of most data. However, the time difference between the trough and peak, expected to be proportional to the thickness of the LVL, is measured as $1.8 \mathrm{~s}$ on this example of standard processing. Using typical $P$ and $S$ velocities for the oceanic crust, this measure would result in a $14 \mathrm{~km}$ thickness of the LVL at the top of the slab. This is twice the $7 \mathrm{~km}$ thickness expected for a standard oceanic crust (White et al. 1992).

Whether the large slab crust thickness thus inferred from standard processing is real or may be due to a resolution problem for thin layers deserves further attention. The phenomenon of wave interaction with LVL inclusion has been identified and taken into account earlier in reflection-refraction investigations of the crust (Fuchs 1969) and uppermost mantle (Hirn et al. 1973; Kennett 1980). The strong dependence of the resulting signal on the scale of fine structure, such as thin LVL embedded in a medium, has indeed been used to identify and quantify such layers. In that case, the source signal was narrow band, but the incidence angles, and thus the apparent thickness sensed by the waves, could be varied by recording reflections at variable offset from the source to large distances. In a simpler way, in the present teleseismic $P$-to- $S$ conversion, or RF approach, the signal from an earthquake source has a much broader spectrum. It provides us with a broad range of signal periods or wavelengths that we may thus use in order to identify and quantify the embedded
LVL by the variation of its response with frequency. We also take advantage of the more recent development of the wavelet transform (e.g. Mallat \& Hwang 1992) that has the property of identifying singularities at various scales and thus quantify the resolution, here in the velocity-depth function.

\subsection{Wavelet response analysis}

We proceed to localize and identify the singularities of interest in the frame of the wavelet transform approach (e.g. Mallat \& Hwang 1992; Holschneider 1995). In order to remotely probe the structure of singularities in the velocity-depth function, Le Gonidec et al. (2002) and Le Gonidec \& Gibert (2007) studied the reflection of acoustic waves from a medium with velocity-depth variations in laboratory experiments. They verified that the 'wavelet-response' of a velocity-depth function, which they obtained experimentally by propagating a wavelet family through a medium, follows the properties of the classic wavelet transform of that function. In this case, we use the $S$ waves generated by conversions of the $P$ teleseismic body waves on their upward propagation through the velocity-depth structure beneath the receivers. This allows thus to approach singularities in the velocity-depth structure by the variation of the response with period, that is across scales.

In theory, the wavelet-transform of a signal, for example a velocity-depth function, is defined as the result of the convolution of this signal by a wavelet family whose elements have same shape but varied periods and amplitudes since they are derived by successive dilatation $a$ of one analysing wavelet. For convenience, traces obtained for different values of dilatation of the wavelet transform or response are commonly assembled side by side in a scalogram as a function of the wavelet period. For our problem of a crustal layer embedded in the mantle, as an illustration anticipating on the next section, a typical wavelet response in $P$-to- $S$ conversion is represented on a scalogram in Fig. 4. The velocity-depth function does not have a single singularity but two successive steps with opposite polarities. In the scalogram in this Fig. 4, the analysis following Mallat \& Hwang (1992) leads to identify the response of these velocity steps in the traces at the largest dilatation or period and then 
follow them through the scalogram to the shortest periods as the amplitude extrema of the wavelet response along two ridge-functions correlated as dashed lines. Taken separately each of these velocity steps is well known from seismological theory to have a response that is constant with respect to the incoming signal whatever period, but their cluster shows in fact a response varying as a function of scale. If the evolutions of the amplitude of the extrema are represented as a function of wavelet periods such as in the usual diagram of $\log _{2}$ amplitude- $\log _{2}$ period, then the slope will show variations. This allows to identify a multiscale singularity, whose signature changes with the observation scale.

When singularities in the velocity-depth function are far enough from one another to appear as isolated, the evolution across wavelet periods of the amplitude of the extremum along the ridge function can also resolve their nature, such as velocity steps, spikes or gradients. As shown by Mallat \& Hwang (1992), and verified experimentally in acoustic wave propagation by Le Gonidec et al. (2002), the amplitude variation is a straight line in a diagram $\log _{2}$ amplitude- $\log _{2}$ period, with a slope $\alpha=0$ for the Heaviside function, that is, velocity step between layers. A negative slope $\alpha=$ -1 corresponds to the velocity spike or Dirac function for the case of a thin-layer embedded in a medium, and a positive slope $\alpha=1$ corresponds to the onset of a ramp function, like at the boundary between a constant velocity layer and one with a velocity gradient with depth. This is because such singularities can be parametrized by a self-similar mathematical function which is solution of the homogeneity equation $s(k t)=k^{\alpha} s(t)$ (e.g. Holschneider 1995). They thus can be identified by their power-law exponent that is the slope of their bi-logarithmic variation of amplitude versus period.

For seismic observations with limited bandwidth, wavelet response analyses have been applied in a version that uses instead of a suite of wavelet of different dilatations or scales, the set of derivatives of a single wavelet on a fixed scale support, such as in reflection seismics for the characterization of seismic facies (Herrmann et al. 2001), or for studying deep upper-mantle transition zones with $S$ converted from teleseismic $P$ (Herrmann \& Bernabé 2004). RF stacks of such deep upper-mantle transitions have also been processed by the wavelet transform using a Morlet wavelet (Castillo et al. 2001). We choose here to make use of the full extent of the frequency spectrum of the source of each particular earthquake, since the teleseismic $P$ signal is often found to extend over more than three octaves. Furthermore, we use the specific suite of wavelets derived from filtering and deconvolving the particular signature of each earthquake, in order to perform a multiresolution analysis.

The RF processing is indeed a deconvolution of the $S v$ component by the $P$ component to get rid of the source time-function for such reasons as for instance that it may be longer than the time interval between the impinging $P$ and its conversions of interest. In practice, we use the procedure implemented by Stammler (1993), in which the inverse filtering of Berkhout (1977) is used. The parameters of the inverse filter adapt to concentrate into a zero-phase wavelet the whole $P$ signal on the $\mathrm{L}$ component. Deconvolution is obtained by applying the corresponding filter parameters to the original $S v$ on the Q-component. The result is the response in conversion to the corresponding $P$ zero-phase wavelet. Since we normalize the wavelets to a same unit amplitude it is the wavelet response of Le Gonidec \& Gibert (2007). This is hence the wavelet transform, to an amplitude factor proportional to scale that is wavelet dilatation (Holschneider 1995). This renders this specific RF estimation procedure akin to the study of the wavelet-response in reflection seismics, whereas the source used there is better known since generated in a controlled way as part of the survey.

As an example of the wavelet analysis in $P$-to- $S$ conversion, the RF response on the $S v$ component as a function of the $P$ wavelet period is computed. In practice, we apply a wide range of different bandpass filters to the original seismogram and we construct the scalogram with the suite of RF resulting from deconvolution, as for example in Fig. 3 for the same Kuril earthquake at VLI as in Fig. 2.

This procedure allows an unprecedented identification of the main feature observed on the figure, composed of a trough followed by a peak and corresponding to the top and bottom of the LVL at the top of the slab in the Hellenic subduction. This feature can be followed with high amplitudes through all periods or scales along the ridge functions in the classical wavelet-transform. Since each ridge function is seen to vary both in arrival time and amplitude across scales, this evidences that the LVL at the slab top has a response in conversion that depends on the period or scale. This illustrates that the interpretation of structure in terms of the LVL depth, thickness and velocity contrast, inferred from RF conversion arrival-times and amplitudes would greatly change depending on the period.

The RF highlighted as bold in Fig. 3 is the same as in Fig. 2, with the commonly used high-frequency cut-off at $0.3 \mathrm{~Hz}$. As mentioned earlier, this standard processing would result in a $1.8 \mathrm{~s}$ trough to peak time interval, yielding $14 \mathrm{~km}$ thickness for a crustal LVL at the top of the slab. It is made obvious from this scalogram that this

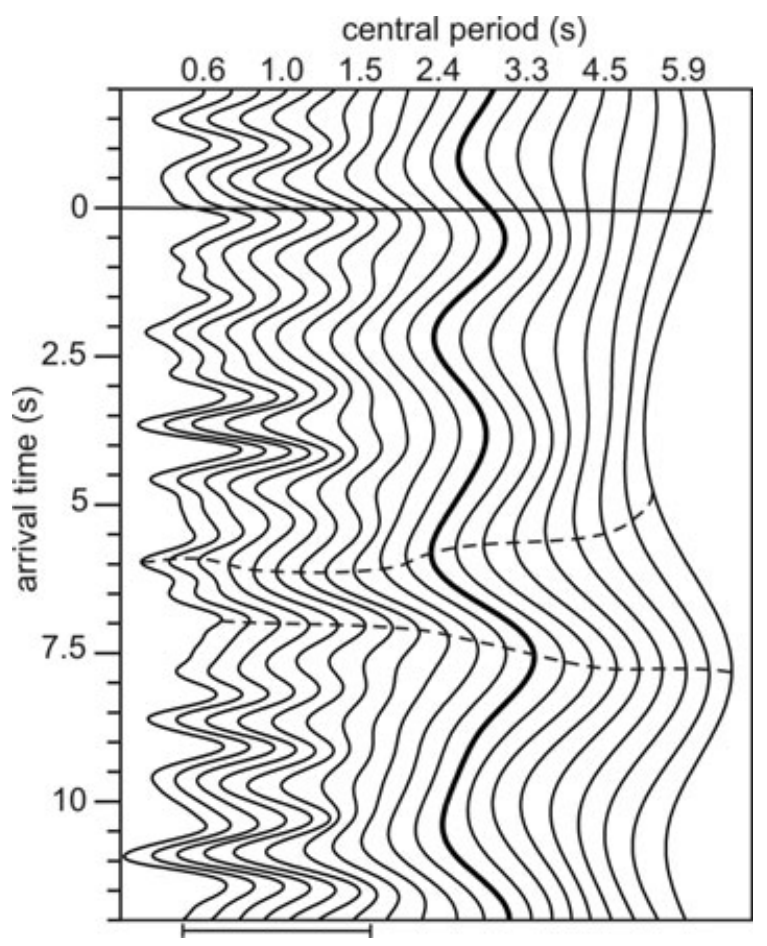

Figure 3. Scalogram of RF, showing the wavelet-response in $P$-to- $S$ conversion of the structure beneath the VLI station. (The segment below the figure gives the amplitude of the corresponding $P$.) Traces for different central periods are obtained by application of filters with different bandpass to the original seismograms of the $M_{\mathrm{w}} \sim 6.6$ Kuril earthquake, followed by the deconvolution step. The amplitude extrema of the wavelet response of the LVL at the Hellenic slab top are followed along ridge functions (dashed lines). Bold highlighted trace corresponds to the RF of Fig. 2. 
estimate has to be greatly decreased by up to nearly half this value, when considering the $P$ analysing wavelet to shorter periods.

In the next part, we develop the theoretical wavelet response in conversion and define resolution criteria for the case of low-velocity inclusions such as typical oceanic crust by computing synthetic RF. We will also validate the whole approach of multiresolution analysis on synthetic seismograms obtained for the case of a dipping slab.

\section{MULTIRESOLUTION ANALYSIS OF THE LOW VELOCITY ZONE RESPONSE IN CONVERSION:SYNTHETIC EXAMPLES}

\subsection{Wavelet response of a low-velocity layer}

We first consider the simplest horizontally layered velocity-depth model of an oceanic crust on top of the slab under the overlying mantle. The wavelet response in conversion (Fig. 4) of a 7-kmthick LVL with $V_{P}=6.5 \mathrm{~km} \mathrm{~s}^{-1}$ and $V_{P} / V_{S}=1.8$, embedded in a mantle-velocity medium at $50 \mathrm{~km}$ depth, is computed for the case of teleseismic incidence, using the forward modelling of Kind et al. (1995) and Stammler (1993). The theoretical delay time between the wave converted at the top and the wave converted at the base of the LVL, which is also between a path in $P$ and in $S$ in this LVL, is

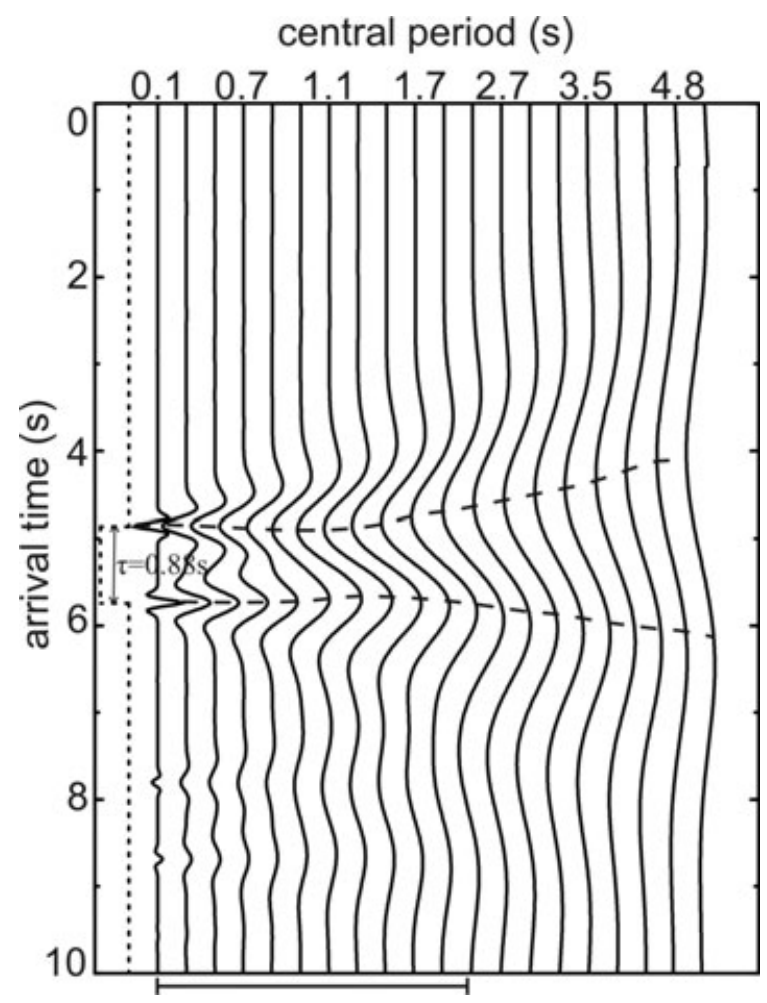

Figure 4. Scalogram showing wavelet response of synthetic RF, computed for a model of an oceanic crust $7 \mathrm{~km}$ thick at slab top (i.e. having a LVL of $V_{P} 6.5 \mathrm{~km} \mathrm{~s}^{-1}$ at $50 \mathrm{~km}$ depth, equivalent to a theoretical time delay of $\tau=0.88 \mathrm{~s}$ as sketched in time as a dotted line on the left) embedded in a medium of $V_{P}=8 \mathrm{~km} \mathrm{~s}^{-1}$, and with $V_{P} / V_{S}=1.8$ throughout. Synthetic RF sorted according to the $P$ wavelet central period $T_{\mathrm{c}}$ (note that this is not the nominal or peak period $T_{\mathrm{p}}$ commonly used in the identification of the Ricker wavelets, but according to their equation $T_{\mathrm{c}} \sim 0.67 T_{\mathrm{p}}$ ). Amplitude extrema are followed along ridge functions (dashed lines). (The segment below the figure gives the amplitude of the corresponding $P$ wavelet.)

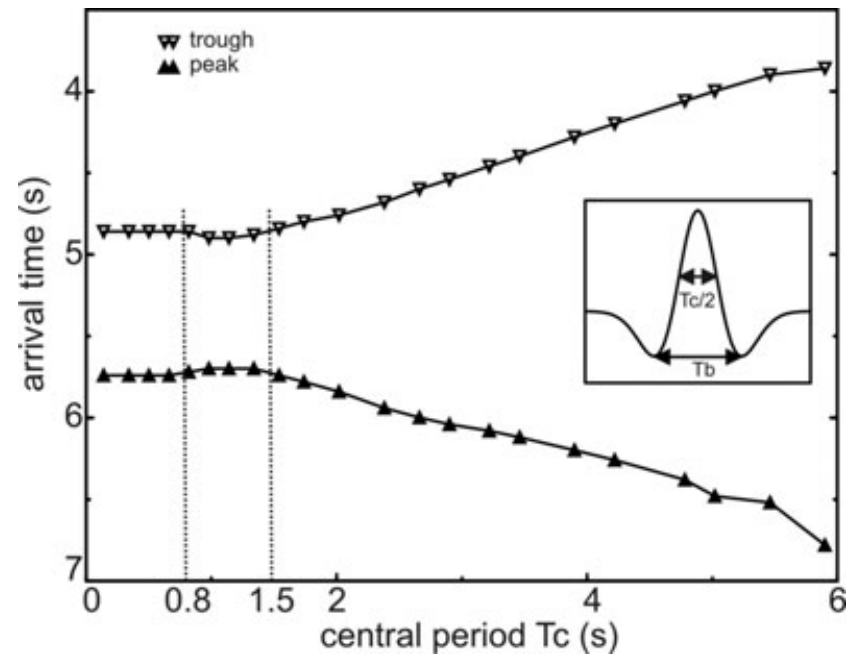

Figure 5. Time attributes of the synthetic RF of the simple model of Fig. 4, with arrival times of the trough as open inverted triangles and those of the peak as full triangles, as a function of the central period of the Ricker wavelet used. Insert: Illustration for a Ricker wavelet of the two definitions of period used in the study: the central period $T_{\mathrm{c}}$ and the dominant period $T_{\mathrm{b}}$.

equal to $0.88 \mathrm{~s}$ (Fig. 4). The computation of the synthetic RF is done using for $P$ a Ricker wavelet family of varying period but constant amplitude. Two measures of period are commonly used for zerophase wavelets (Fig. 5, insert). Following Widess (1973), the central period $T_{\mathrm{c}}$ is defined as twice the time separation between inflection points on the central peak, and the dominant period $T_{\mathrm{b}}$ also called wavelet breadth is defined as the time separation between the two side lobes of the wavelet (Fig. 5, insert). For the Ricker wavelet used in the examples, these two periods can be measured to be, respectively, about the two-thirds and three-quarters of the peak period, which is commonly used to characterize the Ricker, as can also be derived formally from the wavelet being described by a known equation in this particular case.

It is obvious that the response of the modelled subducting oceanic crust, here at about $5 \mathrm{~s}$ time (Fig. 4), changes with the frequency of the incoming signal in terms of arrival times and amplitude of the converted waves. These are the main attributes commonly used to characterize the inclusion: the velocity contrasts at its boundaries are deduced from the amplitudes, its depth is deduced from the arrival time of the trough and its thickness from the spacing between the trough and peak. For this synthetic example, the picked arrival times of the trough and peak are represented with respect to the Ricker wavelet central period. As shown by their significant variation through the scalogram in Fig. 5, they cannot be related in a simple way to the depths of the two boundaries of the inclusion. Only for wavelet periods shorter than about $0.8 \mathrm{~s}$, is the measured spacing between the trough and peak (called $\Delta t$ hereafter) equal to the theoretical time delay (called $\tau$ hereafter). Thus, the true depths and the thickness can be univocally retrieved only in this shortperiod domain, whereas with wavelet periods longer than $T_{\mathrm{c}}=$ $1.5 \mathrm{~s}$ the layer time-thickness gets increasingly overestimated.

Previous studies of thin-layer response in reflection seismics established that the parameter which better characterizes the response of an inclusion is the ratio $\lambda / d$ (e.g. Widess 1973) where $\lambda=T V_{P}$ is the wavelength of the signal and $d$ the thickness of the inclusion. A variation of this parameter can be obtained by a variation of the layer thickness as considered by de Voogd \& den Roijen (1983) or by a variation of the wavelet period as we consider here. In conversion 
upon transmission, the characteristic wavelength $\lambda$ in the inclusion depends on the dominant period $T_{\mathrm{b}}$ of the wavelet and on both $P$ and $S$ layer velocities, by the relation $\lambda=T_{\mathrm{b}} /\left(1 / V_{S}-1 / V_{P}\right)$ (when corrected for the incidence angle). The parameter controlling the response then expresses as $\lambda / d=T_{\mathrm{b}} /\left[d\left(1 / V_{S}-1 / V_{P}\right)\right]$ being also equal to $\lambda / d=T_{\mathrm{b}} / \tau$. The main characters of the wavelet response of the multiscale singularity can be summarized by the study of two signal attributes for varying $\lambda / d$ : the amplitudes of the two converted waves (trough and peak) and the spacing between them.

\subsubsection{Amplitude}

In order to compare our study to the wavelet response of a window function in the acoustic case (Le Gonidec et al. 2002), the amplitude evolution is represented in a $\log _{2}-\log _{2}$ diagram (Fig. 6a) with respect to the dominant period $T_{\mathrm{b}}$ and to the ratio $\lambda / \mathrm{d}$. This representation allows to discuss the geometry of singularities in the velocity-depth function, since the slope of the curve indicates the homogeneity degree of the singularity as recalled earlier (Mallat \& Hwang 1992). Slope variations are observed on the diagram and indicate that the LVL representing an oceanic crust appears not as an isolated singularity but as a multiscale singularity, which is differently seen depending on the signal frequency. Three domains of different evolution of the response with period can be defined according to their slope. Their boundaries correspond to particular values of the ratio $\lambda / d$. For the inclusion of a standard oceanic crust considered here, the domain ' $A$ ' of no-interaction between the wave converted at the top and the one converted at the base from a same incoming wave is defined by $\lambda / d<1$ that is $T_{\mathrm{b}}<$ $0.88 \mathrm{~s}$. The domain ' $\mathrm{B}$ ' of interference is defined by $1<\lambda / d<4$, that is for $0.88 \mathrm{~s}<T_{\mathrm{b}}<3.52 \mathrm{~s}$. The thin-layer domain ' $\mathrm{C}$ ', is for $\lambda / d>4$, that is for $T_{\mathrm{b}}>3.52 \mathrm{~s}$.

In the no-interaction domain $\mathrm{A}$, the amplitude is constant with period and corresponds to the true velocity contrast, (here the same at the top and at the base of the LVL), which can thus be directly deduced. The slope is equal to $\alpha=0$, the characteristic value for the step function, and the signal distinguishes each of the two interfaces which form the boundaries of the LVL. For intermediate periods of the B domain, the amplitude varies due to interferences between the converted waves at the top and at the base of the inclusion, reaching a maximum for $T_{\mathrm{b}} \approx 1.76 \mathrm{~s}$, corresponding to constructive interferences that is to the resonance condition $\lambda / d=2$. This is similar to reflection seismics for which the resonance condition corresponds to $\lambda / d=4$ (Widess 1973) where $\lambda=T V_{P}$ because there is a two-way path in the inclusion. In this domain, the velocity contrast cannot any more be deduced from the amplitude.

This amplitude is much increased from its value in the noninteraction domain, by a quantity as much as 40 per cent depending on details of the wavelet, as tested with different Morlet, Ricker and sinc wavelets. For example, for a dominant period of $1.8 \mathrm{~s}$, the $S$ velocity inferred for the LVL of crust between mantle material would be of $3.4 \mathrm{~km} \mathrm{~s}^{-1}$ instead of the true $3.8 \mathrm{~km} \mathrm{~s}^{-1}$. This results in the LVL appearing with a velocity that, as a function of the signal period considered, can be either of upper or lower crustal value if embedded in a normal mantle, so that amplitude considerations can be misleading when not based on a detailed analysis. Towards longer periods beyond this resonance, in the domain $\mathrm{C}$, the troughto-peak amplitude decreases with a slope equal to $\alpha=-1$, the value characteristic of a Dirac distribution that corresponds to the thinlayer case, as was previously noted in the reflection case (Widess 1973).
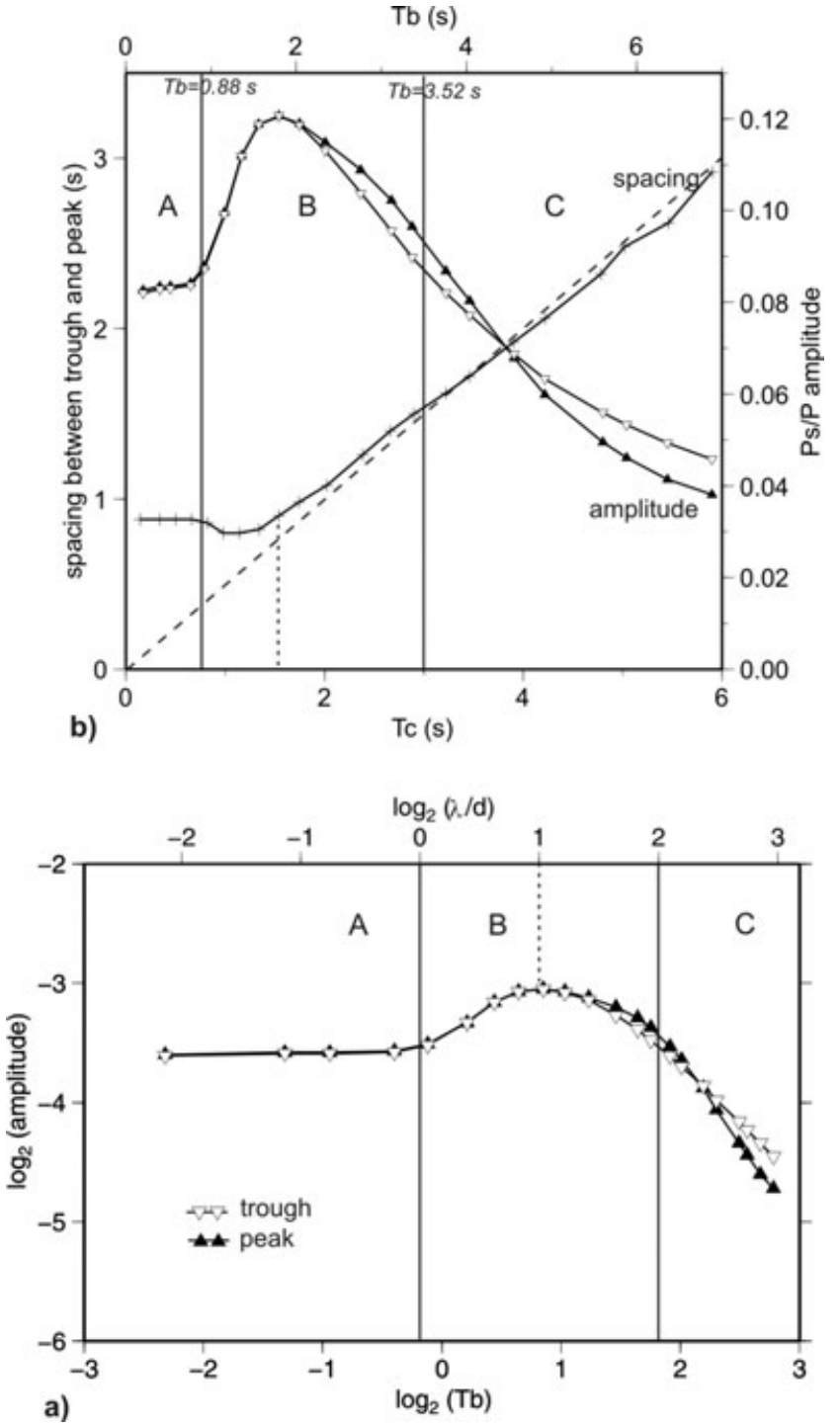

Figure 6. Other attributes of the synthetic RF of the simple model of Fig. 4. (a) Amplitude versus dominant period in a $\log _{2}-\log _{2}$ diagram of the trough (open inverted triangles) and of the peak (full triangles), with respect to the dominant period $T_{\mathrm{b}}$, (lower axis). Upper axis is the ratio $\lambda / d$ (with $\lambda$ the characteristic wavelength and $d$ the thickness of the LVL), with $\log _{2} \lambda / d$ values of 0 and 2 bounding the three domains corresponding to the noninteraction (A), the interference-resonance domain (B), and the thin-layer domain (C). The dotted vertical line indicates $\lambda / d=2$ for which conversion amplitude is maximum (resonance). (b) Time-spacing (scale on the left) between the trough and peak as a function of the central period $T_{\mathrm{c}}$ (lower axis). The dashed line represents the Ricker criterion of limit of resolvability of a thin bed $\Delta t=T_{\mathrm{c}} / 2$. Curves for amplitudes (scale on the right) of the trough (open inverted triangles) and of the peak (full triangles), as a function to the dominant period $T_{\mathrm{b}}$ (upper axis), with resonance for $\lambda / d=2$.

\subsubsection{Arrival times}

Following the variation of the spacing between the trough and peak with respect to the central period (Fig. 6b) allows to analyse more easily the evolution of the apparent thickness of the LVL. In the no-interaction A domain, this spacing is constant and equal to the theoretical time delay $\tau=0.88 \mathrm{~s}$ in the LVL. In the interference B domain, this spacing between trough and peak decreases slightly and then increases strongly with increasing period. The amount of decrease varies with the type of wavelet as tested with several 
Morlet, Ricker and sinc wavelets. This may induce a decrease as large as 25 per cent of the spacing between the trough and the peak, with respect to the non-interference value at shortest periods. In the part of this domain B where this spacing increases again, its value equals the theoretical time delay of the no-interaction domain, for the particular value of $T_{\mathrm{b}}=1.76 \mathrm{~s}$ (i.e. $\Delta t=\tau=T_{\mathrm{b}} / 2$ or $\lambda / d=2$ ). This value of period is where amplitudes are also most increased by resonance, with respect to the no-interaction domain. This condition corresponds to the Rayleigh criterion which states that the resolution limit between two source objects occurs when the maxima of their two diffraction patterns are separated by half the distance between the minima of the two side lobes of one diffraction pattern (e.g. Widess 1973). This condition also corresponds to the tuning thickness in reflection seismics (Kallweit \& Wood 1982) where apparent thickness is the same as true thickness.

In the thin-layer domain $\mathrm{C}$ for $T_{\mathrm{b}}>3.52 \mathrm{~s}$, for which the LVL corresponds to a spike in the velocity-depth function with respect to the period of the incoming wavelet, the response has the shape of the derivative of the $P$ wavelet, that is with one more extremum, as described by Widess (1973) in reflection seismics. Indeed this means that the LVL, when seen as a spike by a long period wavelet has still a response with a trough and peak because the orders of homogeneity of the velocity step and spike differ by one. Obtaining the trough and peak signature is thus not diagnostic of the LVL thickness being resolved or not. The limit of resolvability of a thinbed according to the Ricker criterion (Widess 1973) occurs when two zero-phase wavelets are separated by an interval equal to the time separation between the inflection points on their central peak. For longer wavelet periods, the trough to peak spacing in the RF caused by the LVL that is seen as a spike, increases then linearly with the incoming $P$ wavelet period and is simply equal to $\Delta t=T_{\mathrm{c}} / 2$. For incoming $P$ wavelet periods longer than $T_{\mathrm{b}}=1.76 \mathrm{~s}$, which is over twice the no-interference value of the trough-to-peak spacing of the $\mathrm{RF}$, the trough-to-peak values observed have lost any relation with the characteristics of the LVL, which conversely cannot be deduced from the observed spacing.

In summary the normal 7-km-thick oceanic crust can be only measured directly by the trough-peak spacing and amplitudes for wavelets with a central period shorter than about $0.8 \mathrm{~s}$, this upper limit slightly depending on the type of wavelet. For the typical oceanic crust and for a signal period of $T_{\mathrm{c}}=3 \mathrm{~s}\left(T_{\mathrm{b}}=3.5 \mathrm{~s}\right)$ resulting from commonly used filtering with $0.3 \mathrm{~Hz}$ low-pass filter, Fig. 6(b) illustrates that a trough to peak time of $1.5 \mathrm{~s}$ would be found that is a thickness of $12 \mathrm{~km}$ would be inferred, which is almost twice the true thickness of the oceanic crust in the model.

\subsection{Analysis of numerical simulation of propagation and conversion in realistic model in section of dipping slab}

The previous example on the synthetic RF response shows that multiresolution analysis in conversion can characterize inclusions at depth such as a LVL at the slab top. In order to validate and refine the strategy to be applied in the analysis of real data, we need to check two aspects.

First, we have to estimate the effect, with respect to the forward modelling with a wavelet family to obtain the scalogram as in Fig. 4, of the RF processing which we apply to really observed seismograms to obtain a scalogram for observed signals, as in Fig. 3. In order to do this check, we will compute full waveform synthetic seismograms resulting from the propagation through a known model for a source with a broad but still limited frequency band, and apply to them the same RF processing as for real data.

Second, we have to check the effect of a structural dip, such as is likely for the slab top, with respect to the synthetic responses for a 1-D velocity-depth function considered before. Therefore we introduce such a dipping slab crust into the 2-D velocity-depth model.

In order to check these aspects over a wide band of periods, we use two sources each of 3 octaves-broad spectrum with dominant periods respectively $T_{\mathrm{b}}=0.64 \mathrm{~s}\left(T_{\mathrm{c}}=0.6 \mathrm{~s}\right)$ and $T_{\mathrm{b}}=1.68 \mathrm{~s}$ $\left(T_{\mathrm{c}}=1.55 \mathrm{~s}\right)$. A set of synthetic seismograms is obtained by a full waveform simulation implemented and run by D. Rousset at Pau University using finite differences (Virieux 1986). The $P$ wavefront, with the incidence from beneath of a teleseismic wave, is propagating updip in the $2-\mathrm{D}$-section of a model containing a $45^{\circ}$ dipping, 10-km-thick oceanic crust. This is embedded between a forearc mantle and a slab mantle given the same velocity. The value of the dip, stronger than expected for the Hellenic slab, is chosen to make any effect more obvious. The thickness of the layer corresponds to the upper limit of that expected for an oceanic crust. The wavelet analysis is performed in exactly the same way as on real data. The original multicomponent seismograms are filtered with different bandpass and from each output the RF is computed by the procedure of Stammler (1993), obtaining for $P$ a zero-phase wavelet. From the three octaves-broad signal, RF can be stably derived by variable bandpass filtering for central periods covering an octave, here $T_{\mathrm{c}}=1.3-2.7 \mathrm{~s}$, around the dominant period. The resulting scalogram, as seen in Fig. 7 for the source with dominant period at $T_{\mathrm{b}}=1.68 \mathrm{~s}$, allows to follow the evolution of waves converted at the top and bottom of the slab crust along the ridge functions (dotted lines) across scales. It appears notably that for the two shortest periods or smallest scales, the sidelobes of the two opposite polarity wavelets associated to the limits of the LVL are still distinguishable whereas for the longer periods they merge by interference. The spacing between the trough and peak is represented with respect to central period (in black in Fig. 8a) for the two sources. Its behaviour can be compared with that in the previous simple 1-D model direct computation of the RF for the Ricker wavelets (Fig. 6b). Very similarly, there is here a domain A of constant spacing for shorter periods giving the real theoretical time delay in the dipping LVL, then the spacing first decreases then increases again, passing over the value at the shortest periods for $\lambda / d=2$, and continuing to increase for longer periods. The amplitude of the relative decrease of the spacing in the domain B with respect to A had been already seen in the case of horizontal structure to have different magnitudes with the different specific wavelets used, here it is as much as 20 per cent.

In order to take into account the effect of the possibly variable shapes of the $P$ wavelet obtained by filtering of the signal, we use a simple procedure. We compute synthetic RF through a 1-D model but we use as input the suite of the $P$ wavelets derived from the different filter settings on the synthetic seismograms obtained by propagation through the $2-\mathrm{D}$ model. In the $1-\mathrm{D}$ model the LVL is given a $11.8 \mathrm{~km}$ instead of $10 \mathrm{~km}$ thickness to compensate for the effect that propagation is now not anymore updip. The resulting green curve of the spacing between trough and peak on Fig. 8(a) retrieves almost exactly the black curve obtained from processing the seismograms of the propagation in the 2-D model. Hence the evolution of spacing appears not to be affected by the dip, when using the apparent LVL thickness compensated for it.

The coincidence in detail of the two spacing-period curves illustrates also another important aspect. It shows that when taking as 


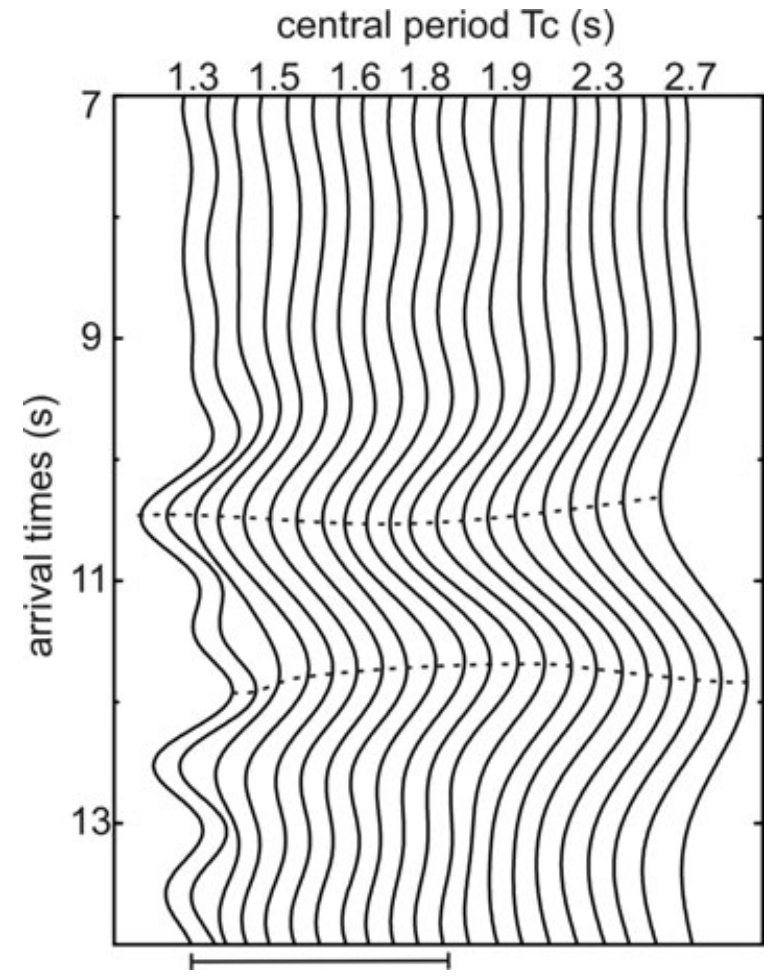

Figure 7. Scalogram analysis of RF processed from a full-waveform multicomponent seismogram obtained numerically from propagation in a realistic model of a 2-D section of a slab having a $45^{\circ}$ dipping 10-km-thick LVL embedded in a medium of mantle-like velocity with updip incidence from below. Source signal has $1.55 \mathrm{~s}$ central period and three octaves bandwidth. $\mathrm{RF}$, represented here for arrival times between 7 and $13 \mathrm{~s}$, are obtained as for observed data by application of filters with different bandpass to the full waveform synthetics, followed by the deconvolution step. Dotted lines represent the ridge functions. (The segment below the figure gives the amplitude of the corresponding $P$ wavelet.)

input to the 1-D synthetics the $P$ wavelets that do not have strictly the same shapes, as a result of filtering the signal, a very fine agreement is obtained with the RF processing of the result of the real propagation of the bandlimited signal. The effects which could be due to their deviation from strict similarity in shape can thus be obviated in practice.

The amplitudes of trough and peak are represented with respect to dominant periods (in black in Fig. 8b). The no-interaction domain is seen through the range of wavelets extracted from the source signal with shorter dominant period by their rather constant amplitude response, with a zero slope in the $\log _{2}$ amplitude- $\log _{2}$ period diagram. The interference domain is seen through the range of wavelets extracted from the longer period source, which shows an amplitude maximum that has its slope varying from positive to negative around resonance. With respect to these variations, the amplitude level itself is dominated by the effect of dip. The amplitudes measured from the propagation in the medium accounting for the slab dip (in black in Fig. 8b) are clearly higher, twice the value computed for a horizontal slab (green symbols in Fig. 8b). Shifting amplitude up by taking the theoretical effect of dip on them into account (green dotted lines) then reproduces quite well the amplitude evolution of the RF of synthetic seismograms. Apart this amplitude level, the clearest difference with respect to the direct RF synthetics computed in the previous section with horizontal layering and Ricker wavelets is a small variation of the relative amplitudes of the trough

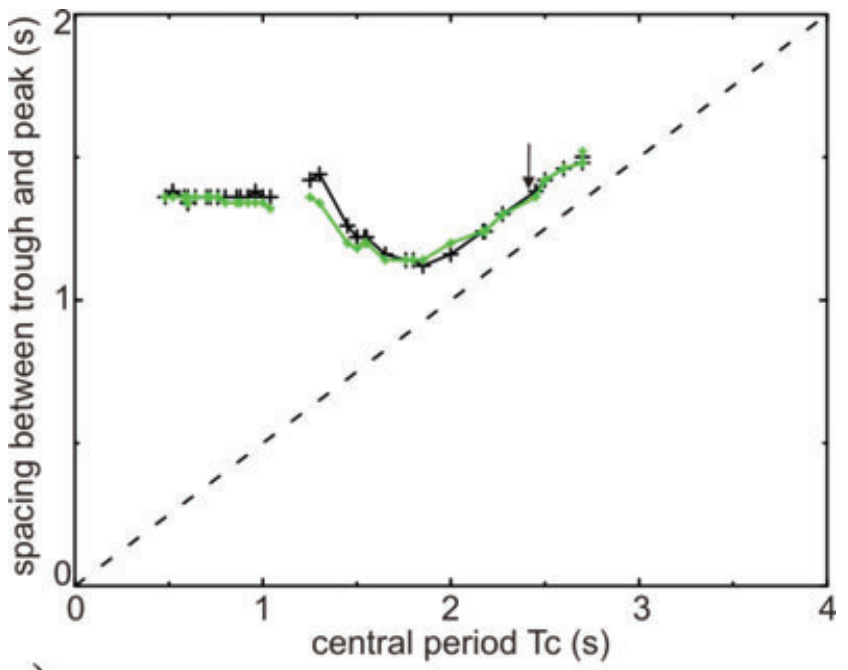

a)

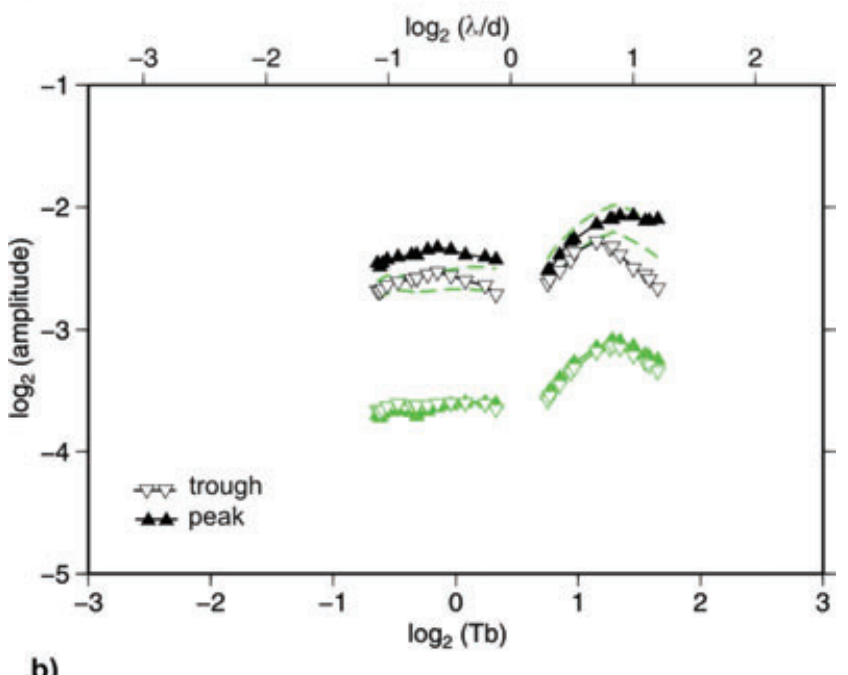

b)

Figure 8. Analysis of the scalogram of Fig. 7 of RF resulting from processing of full-waveform synthetics in the dipping slab, for sources of $1.55 \mathrm{~s}$ and of $0.6 \mathrm{~s}$ central periods. In black, spacing between trough and peak (a) and their amplitudes (b) with respect to the signal period. In green, spacing and amplitudes of synthetic RF in a horizontally layered model using as an input the $P$ wavelet obtained after each bandpass filtering and deconvolution of the full waveform synthetic seismograms. For the amplitudes in (b) the green dotted lines are derived from the full lines by taking into account the theoretical amplitude increase due to the updip propagation with respect to the slab. The arrow on (a) indicates the point for which $\lambda / d=2$ where the spacing between trough and peak is equal to the real time delay in the dipping layer as expected.

and of the peak. This can be attributed to the dip, as a consequence of the incidence angle of the $P$ wave on the interfaces being different below the LVL and after propagating through it, at its top. The maximum of the peak is found as expected at $\lambda / d=2$.

Principal characteristics expected for a LVL are retrieved from the analysis of the wavelet response applied to full waveform synthetic seismograms and validate this approach for the case of real data. Applying filters of different bandpass to seismograms prior to the deconvolution step permits conducting a multiresolution analysis and identifying the characteristics of a LVL at depth such as a slab crust. 


\section{WAVELET ANALYSIS OF REAL DATA: OCEANIC SLAB, $180 \mathrm{~km}$ INBOARD THE SUBDUCTION BACKSTOP}

\subsection{Avoiding an underestimation of the thickness: earthquake source dependant effects}

At the same location, with the same system of seismometer and recorder, and seen through the same bandpass filter, the incoming signals of different earthquakes do not produce the same $P$ wavelet shape in detail, not even with the same central and dominant periods. This is even true for neighbouring earthquakes, for which propagation occurs along the same path. There is thus clearly an effect of the particular spectrum of each different source.

We have searched the database for examples illustrating the reported phenomena, and that would allow to discuss and to constrain the real structure.

For larger earthquakes the higher overall amplitude level might correspondingly keep signal above noise down to the shortest periods. This appears to be the case of the example of the VLI record of Fig. 3 obtained in the quieter site of a permanent observatory station. Indeed this particular earthquake has been chosen among several Kuriles earthquakes of about same hypocentral region because significant signal could be recovered down to the bandpass of 0.1 to $1 \mathrm{~s}$, giving a $P$ wavelet with a 0.6 s central period.

Here, the trough-to-peak spacing of the LVL conversion decreases for the $P$ central period decreasing from $T_{\mathrm{c}}=6 \mathrm{~s}$ to $1.25 \mathrm{~s}$, but as it can be followed to a $P$ period as short as $T_{\mathrm{c}}=0.6 \mathrm{~s}$, it is seen to increase again slightly, then stabilize. This behaviour is similar to the variation obtained in the synthetics of Fig. 6 that have allowed the discussion of the domains of interference A, B and C. Indeed this spacing decreases from long periods and its reversal for the shortest allows to define when the non-interfering value of time-thickness is reached.

We applied the wavelet analysis to the AGBA station located about $50 \mathrm{~km}$ to the North of the VLI station. The wavelet response (Fig. 9) of a Honshu earthquake (2008 July 19, $M=6.9$, backazimuth $44^{\circ}$, distance $87^{\circ}$ ) shows again variations. A wide spacing of about $1.7 \mathrm{~s}$ between the trough and peak is again obtained with the commonly used $0.3 \mathrm{~Hz}$ high-frequency cut-off, giving here a corresponding central period of $T_{\mathrm{c}}=3.1 \mathrm{~s}$. This would suggest a thickness as large as $15 \mathrm{~km}$ for the crust of the slab. This thickness estimate can be shown as inadequate since the trough to peak delay can be greatly reduced when decreasing signal periods. Interestingly, an even very thin signature is evidenced at the shortest period obtained for this event (here $T_{\mathrm{c}}=0.8 \mathrm{~s}$ ), $\Delta t=0.75 \mathrm{~s}$ which would correspond to less than $6 \mathrm{~km}$ of oceanic crust. The shortening of the signature to values smaller than the time delay in the LVL is predicted by the synthetic seismograms modelling (Section 5.2, or Fig. 8a).

These examples show that if the interference effects due to the frequency are not taken into account, two opposite types of wrong interpretations can be reached. A standard processing with a $0.3 \mathrm{~Hz}$ high-frequency cut-off will largely overestimate the LVL thickness, such as by a factor 2 . On the other hand, a higher frequency processing may lead to an underestimation of the thickness, if the shortest period available from the particular earthquake would remain in the interference domain. Hence, very high frequency signals are necessary to capture the change from the interference domain, where the spacing underestimates the inclusion layer thickness, to the noninterference domain of the converted waves, and then propose a documented reliable structural interpretation.

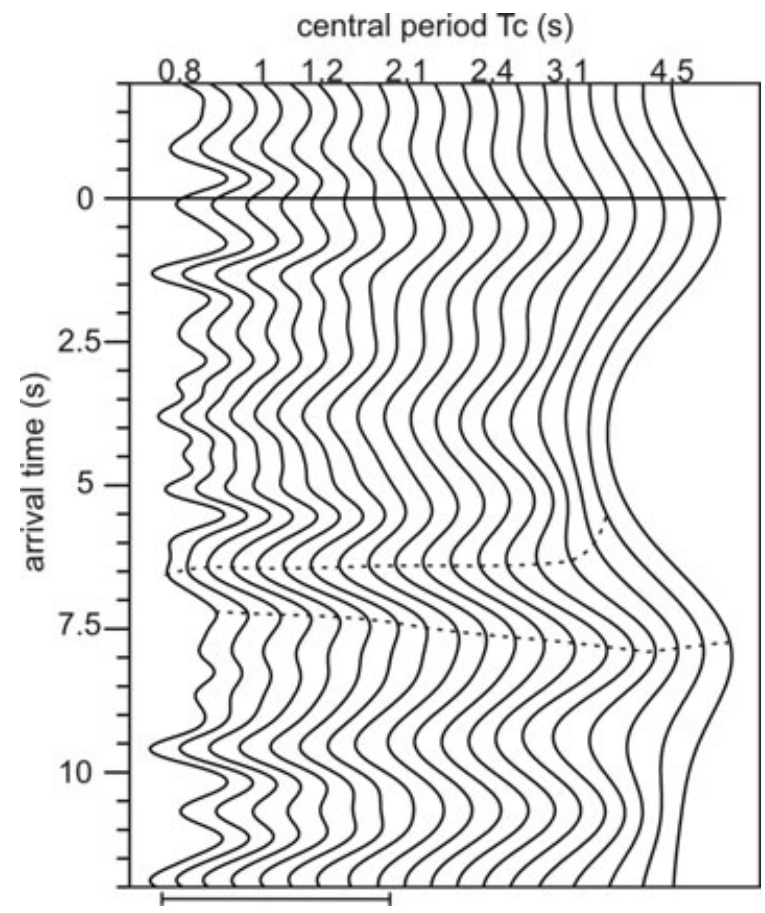

Figure 9. Scalogram of RF, or wavelet response in conversion, of the structure beneath the AGBA station (Fig. 1) obtained after application of filters with different bandpass to the original seismograms of a Honshu earthquake (2008 July 19 , backazimuth $44^{\circ}$, distance $87^{\circ}$ ). Amplitude extrema of the wavelet response of the LVL at Hellenic slab top are followed along ridge functions (dotted lines). (The segment below the figure gives the amplitude of the corresponding $P$ wavelet.)

Deployment since $3 \mathrm{yr}$ of a number of stations allows, with respect to more current shorter deployments or time spans analysed, to compare the results obtained from different source signals and increase the chances of recording unusual events.

Several major earthquakes that could be recorded from each of such source regions as Sumatra, Honshu or Kuriles have provided data, the standard processing of which resulted in RF that on closer analysis showed differences among them for a same propagation path. Hence, diverse interpretations could have been reached in the case only one or few would be available, or if they would have been stacked such as to reduce noise, instead of their differences being analysed and understood. Indeed it appears that with a same standard filter, different earthquakes from neighbouring source locations may result in $P$ wavelets that may differ in diverse aspects, even by over 20 per cent in their resulting wavelet periods. Then, different spacings of the RF trough and peak may result, and thus apparently different values for the LVL thickness sampled at a same location, that will be averaged out to the largest if stacked together.

Having thus established the need for shortest period signal, we searched for very deep sources with a large magnitude. They produce a rich spectrum at short period due to the large depth confinement, and their high amplitude may overcome the effect of attenuation and thus be recorded far away. Over the last $10 \mathrm{yr}$, only 4 earthquakes deeper than $300 \mathrm{~km}$ had magnitudes over 7.5. The major important magnitude $M \sim 7.7$ earthquake at the very large depth of $615 \mathrm{~km}$ under the Sea of Okhotsk on the 2008 July 5 is thus an exceptional event captured in our 3 yr deployment. As illustrated by the RF of the seismograms recorded for instance at the AGBA station (Fig. 10c) the LVL at the slab top has a clear signal to the 

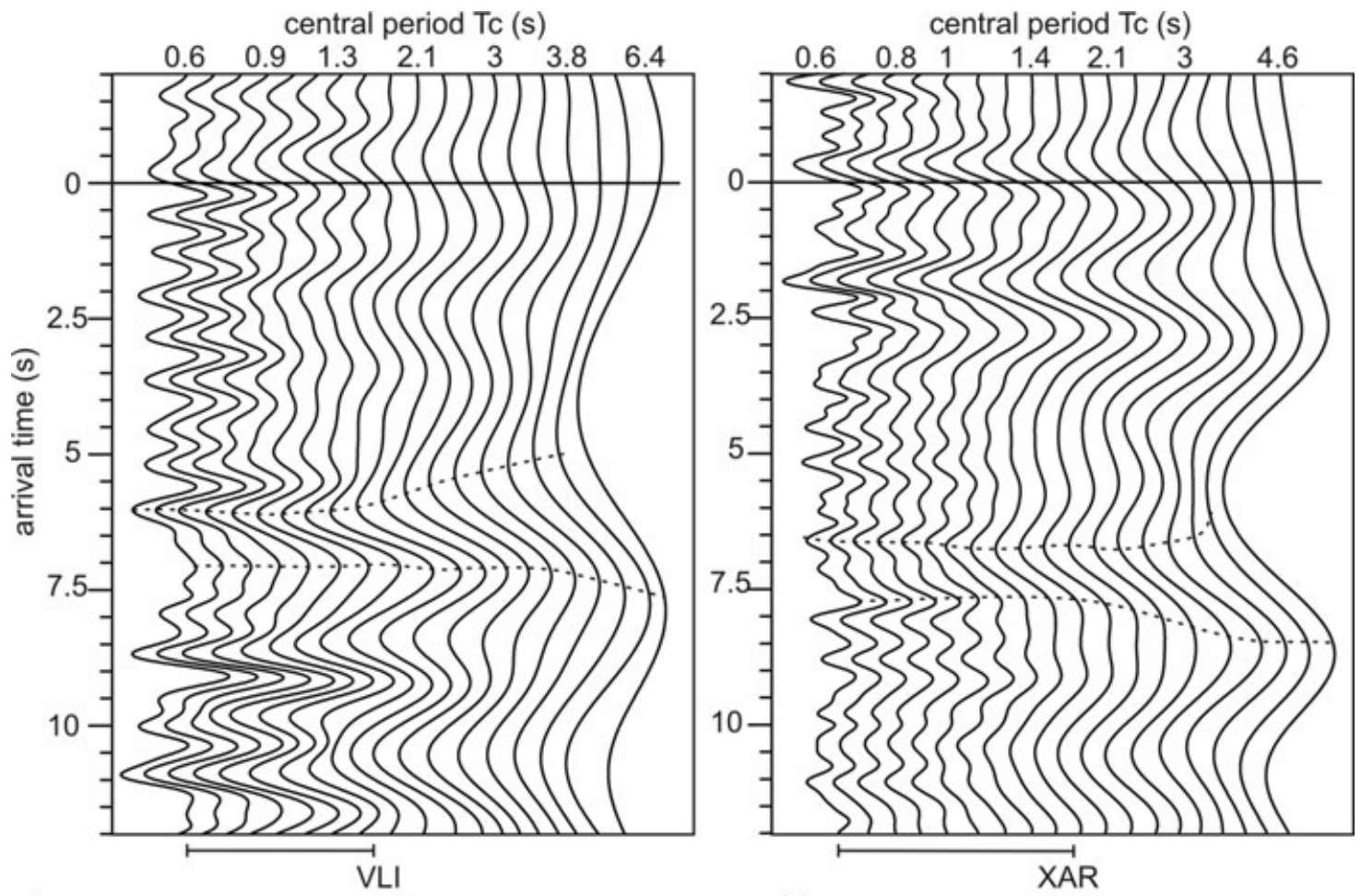

a)

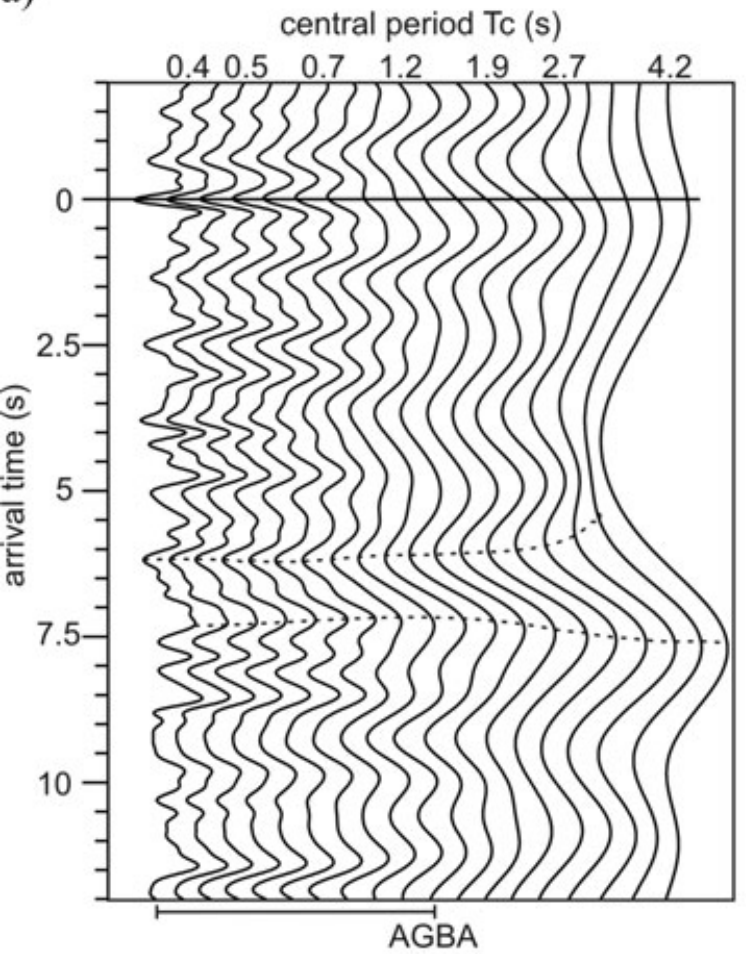

b)

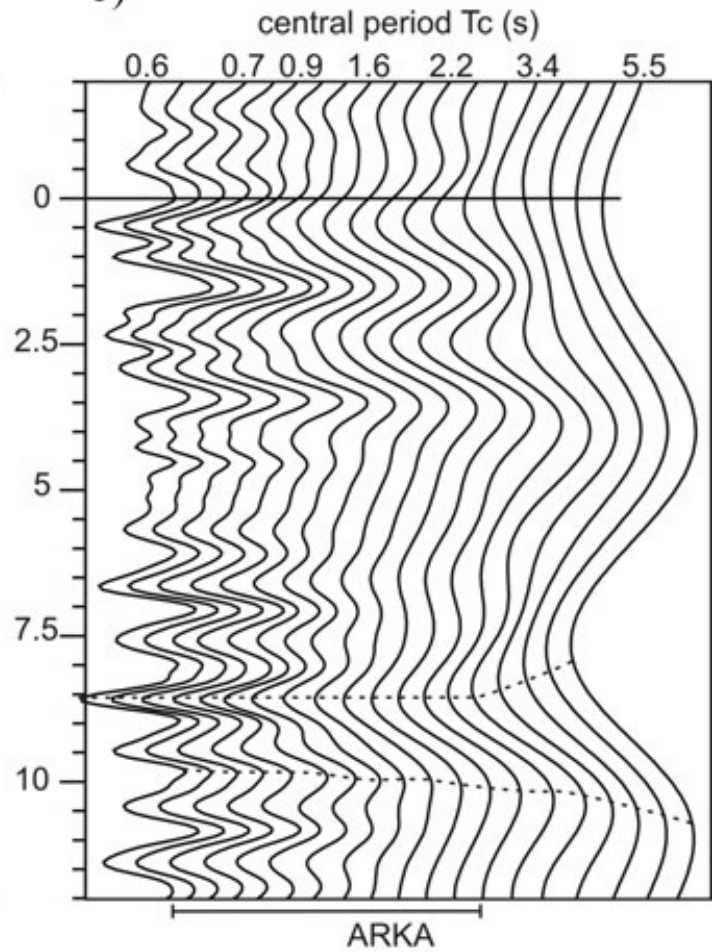

c)

d)

Figure 10. Scalogram of RF, or wavelet response in conversion of the structure beneath stations VLI, XAR, AGBA and ARKA obtained for the original seismograms of the major, over $600 \mathrm{~km}$ deep hence high-frequency earthquake of the Sea of Okhotsk (2008 July $5, M \sim 7.7$, backazimuth $27^{\circ}$, distance $80^{\circ}$ ). Amplitude extrema are followed along ridge functions (dotted lines). (The segment below the figure gives the amplitude of the corresponding $P$ wavelet.)

trace of shortest central period, which is here as short as $T_{\mathrm{c}}=0.42$ $\mathrm{s}$. The corresponding dominant period of $T_{\mathrm{b}}=0.6 \mathrm{~s}$ implies that this earthquake can resolve LVL as thin as $5.5 \mathrm{~km}(\lambda / d<1)$. This occurrence is then a great opportunity to approach the real thickness of the LVL at the top of the Hellenic slab.

\subsection{Wavelet response in southeastern Peloponnesus: an oceanic slab crust}

The wavelet responses derived for the high frequency, large depth and large magnitude Okhotsk earthquake show a clear signature of 
the Hellenic slab top as shown beneath southern Peloponnesus by four stations from south to north along the eastern coast displayed in Fig. 10: VLI, XAR, AGBA and ARKA. The cone-like shape of the variation of the trough and peak pair of extrema in the scalograms indicate strong and wide responses at longer periods. Following the pair of extrema in continuity to short period illustrates the narrowing of their spacing in time and depth, thus more accurately and correctly localizing the slab top. Note that the amplitude levels at short period in Fig. 11(b) of the observed seismograms of Fig. 10 are comparable although scattered, on the order of $\log _{2}$ amplitude $=-3$, that is $1 / 8$ of the $P$ amplitude. They are thus significantly higher than for the short-period response of a typical oceanic crust without dip in the synthetics of Figs. 6(a) or 8(b) (green) but smaller than the amplitude obtained by the processing of the seismograms propagated through the model with a $45^{\circ}$ dip in Fig. 8(b) (black). Amplitudes are thus in agreement to first order with a normal oceanic crust on top of a slab with an intermediate dip on the order of $25^{\circ}$. The dip is certainly less than $45^{\circ}$, and close to $20^{\circ}$, when taking the ratio between the slab depth and the distance from the subduction front or also measuring dip from the azimuthal arrival time variation of the converted waves (Gesret 2008). For the longer periods, these observed amplitudes do not decay as strongly as for the synthetic case of a model containing only a LVL (Fig. 6a). This may be because in the observed data, at the corresponding $T_{\mathrm{b}}$ of $4 \mathrm{~s}$, such a great length of the seismogram is involved that it contains not only the signature of the LVL by itself but the real velocity structure in tens of kilometres of depth around it.

The trough-to-peak spacing value obtained in these observations (Fig. 11a), hardly larger than $1 \mathrm{~s}$ is then consistent to better than $1 \mathrm{~km}$ with a standard 7-km-thick oceanic crust with a $25^{\circ}$ dip. This is when compared to the $0.9 \mathrm{~s}$ value for the trough to peak spacing for a horizontal inclusion of a normal oceanic crust in Section 5.1 and the 20 per cent increase in apparent thickness due to updip propagation for a $45^{\circ}$ dipping slab described in Section 5.2. We have thus constrained both the velocity contrast and thickness as typical of the oceanic crust of the dipping slab, in the $60-80 \mathrm{~km}$ depth range, $150 \mathrm{~km}$ inboard of the subduction backstop boundary. This oceanic value for the thickness of the crust of the slab deduced from the spacing between the trough and peak is an upper limit. This is because, with respect to the normal oceanic crustal velocities used for this estimate, only higher value of $V_{P} / V_{S}$ could have to be considered for geological reasons, such as in the case of hydration in the LVL, but then an even thinner layer estimate would result from this spacing. The wavelet response we developed shows here unambiguously for the first time that the slab presently underlying southeastern Peloponnesus is made of lithosphere with a typically oceanic nature. The arrival time of the trough gives a first order indication on the depth of the top of the LVL at the slab top and its variation, which are consistent through the station network. Indeed the converted wave at the upper boundary of the crustal inclusion that is at the slab top, arrives at $6 \mathrm{~s}$ at VLI (Fig. 10a), at $6.2 \mathrm{~s}$ at AGBA (Fig. 10c), at $6.6 \mathrm{~s}$ at XAR (Fig. 10b) and at $8.6 \mathrm{~s}$ at ARKA (Fig. 10d). This deepening from about 55 to $80 \mathrm{~km}$ is in accordance with a slab dipping in-between to northeast.

Previous studies with the RF method (Li et al. 2003; Sodoudi et al. 2006) report converted waves they detect after that of the Aegean Moho under the station as being generally a single positive pulse, which they interpret as the Moho of the subducted African slab. We obtain instead a waveform that is clearly diagnostic of the top of the slab, with a negative pulse followed in the next second or so by a positive one, hence unmistakably corresponding to the interplate boundary or top of the crust of the slab lithosphere and respectively

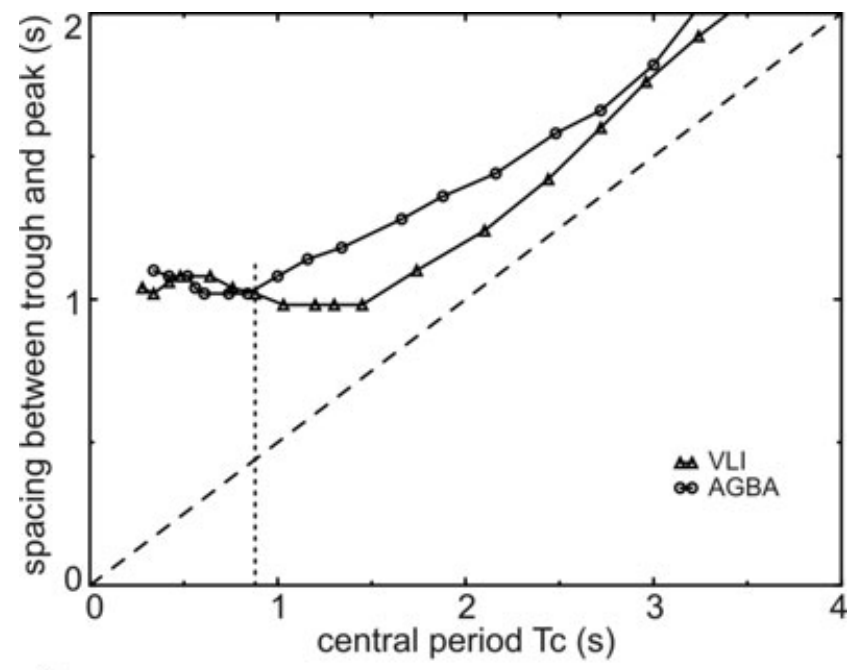

a)

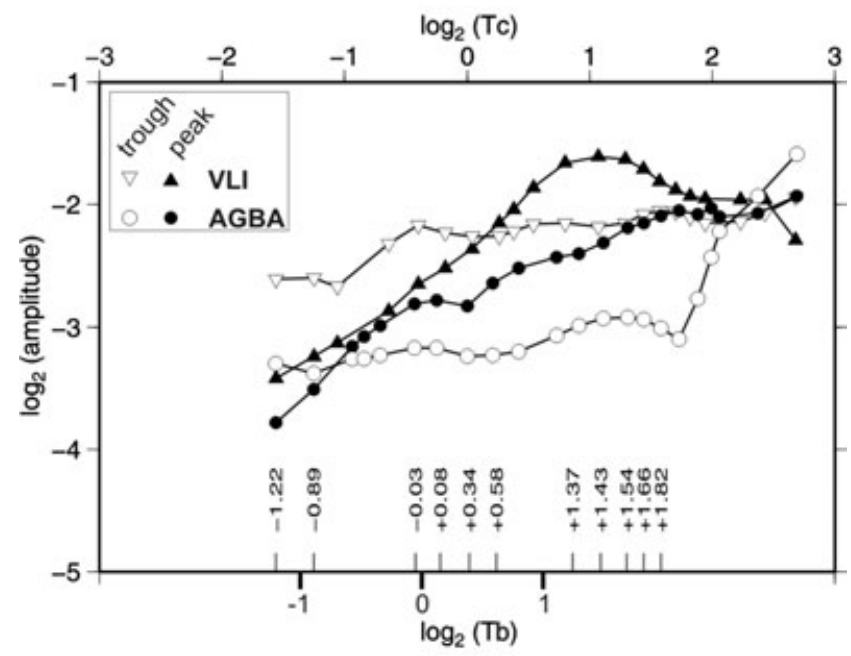

b)

Figure 11. Examples of variation of the spacing and amplitude attributes of the RF of the observed data of the Okhotsk earthquake for station VLI (triangles) of Fig. 10(a) and for station AGBA (circles) of Fig. 10(c), (a) Time-spacing between the trough and peak of the RF with respect to the central period of the $P$ wavelet. The dashed line represents the Ricker's criterion of limit of resolvability of a thin bed $\Delta t=T_{\mathrm{c}} / 2$. The dotted line indicates the period $\left(T_{\mathrm{c}} \sim 0.9 \mathrm{~s}\right)$ above which the spacing varies and cannot be used anymore to determine the thickness of the LVL at the slab top. (b) Amplitude as a function of period for the trough (open symbols) and the peak (full symbols) in a $\log _{2}-\log _{2}$ diagram. Central period $T_{\mathrm{c}}$, on the upper horizontal axis and on the lower one, corresponding values of the dominant period $T_{\mathrm{b}}$ when they could be measured from unambiguous sidelobe separation. This allows comparison with other such diagrams in Figs 6(a) and 8(b).

to the base of this slab crust less than $10 \mathrm{~km}$ deeper. We have conspicuously identified a very strong negative pulse, documenting the subduction interplate boundary. With these two opposite polarity signals we thus obtained quantitative data for a discussion of the slab crust and nature.

Whether the lack of resolution of normally thin oceanic crust in several studies could be due to low frequencies classically used is an open question. Indeed, signals are most often not considered at short period since they are filtered to suppress high-frequency 
noise. From the examples shown here, it appears that the standard processing of the observed data likely leads to see the LVL only in the interference, that is thin-layer, domain, where the trough-peak spacing has lost the relation with the LVL thickness. For studies that use instead $P$ converted from $S$ waves, the situation is even more extreme. The converted $P$ being of the same low-frequency as the $S$ they are converted from, the signal has much lower frequency than $P$ propagated from the earthquake because of both the source radiation and propagation attenuation that are shaping $S$ to long period. Whereas they are useful to map gradational velocity changes at large scale to which they are more sensitive, they are even much worse than $P$-to- $S$ conversions for resolving velocity interfaces in a layered medium. This effect degrades the resolution power of $P$ converted from $S$ with respect to $S$ converted from $P$ in a layered medium, such that they may be blind to the thin LVL inclusion of the lower plate crust between the mantle of the lower plate and the mantle above.

\section{SUMMARY AND CONCLUSIONS}

In the Hellenic subduction zone, the lithospheric slab may comprise continental and oceanic units juxtaposed downdip and along strike, as evidenced from the diversity in the nature of their covers that have been accreted to form the Hellenic nappes at outcrop. Present fast trench retreat, attested by the southwestward fast motion of the edge of the Aegean upper plate domain documented from GPS, would be consistent with the subduction of a purely oceanic slab of most reduced buoyancy of the Ionian sea basin. If this occurred indeed since 4-5 Ma, the slab underlying now eastern Peloponnesus to about $180 \mathrm{~km}$ from the backstop tip should show correspondingly a thin oceanic crust at its top under the overlying mantle, which our objective is to test.

RF processing of teleseismic $P$ waves in the standard frequency band, for stations along eastern Peloponnesus, results in the image of a LVL at the top of the slab twice thicker than expected for the crust of an oceanic slab. Such a large thickness for the LVL between the slab mantle and the overlying mantle has been found in other scattered or converted wave studies, and led to developing new hypotheses on the nature or evolution of subducting slabs.

In such studies elsewhere that found a thick LVL at the top of the slab, this has been considered as an evidence of either one among the three following cases. The first case is that of the subduction of a lithosphere with a thick crust, such as part of a continental crust, an oceanic plateau or an exotic terrane, as in Alaska (Ferris et al. 2003; Rondenay et al. 2008). The second case is the presence of serpentinized mantle exhibiting crust-like velocity beneath the subducted oceanic crust and therefore adding to it for an apparently thicker LVL. This serpentinization might have occurred in its early history, or when the plate enters subduction due to its bendingfaulting and hydration from the top (Ranero et al. 2003). The third case is that of an additional thickness of low-velocity material above the slab. This would be progressively generated by the dehydration of the slab on its way to depth and the related upward fluid migration that would cause progressive serpentinization of the mantle wedge (Kawakatsu \& Watada 2007).

Finding a thick LVL would exclude a strong negative buoyancy of the slab only in the two first cases, since the slab will have in its upper part a thick crust with low-density material, or a mantle with lowered density. In the third case, finding a thick LVL would appear to be inconclusive in disproving an oceanic slab of strong negative buoyancy since the material with lower density is above the slab top. This third hypothesis may however not need to be considered. Indeed, antigorite which is the particular serpentinite produced at large depth has recently been reported to keep a normal mantle-like high velocity and low $V_{P} / V_{S}$ up to a high serpentinization degree (Reynard et al. 2007; Christensen 2004). If this were so, even if an alteration of part of the forearc mantle is present above the slab top, it could thus hardly contribute to thicken the LVL. Hence, finding a thick LVL could only correspond to the first two cases, and thus exclude a negative buoyancy of the slab.

It follows from this discussion that the thick LVL apparently found with standard processing, like in several cases elsewhere, would not be consistent with a subducting slab having strong negative buoyancy, that is of oceanic nature, under Peloponnesus.

In order to assess if this result could be due to a lack of resolution of the standard processing, we developed a multiscale approach with the RFs. It is based on the wavelet-response of the medium, akin to the wavelet-transform of the velocity-depth function. The synthetic response in conversion of a multiscale singularity formed by two opposite velocity-steps at the boundaries of a crust embedded in mantle material leads to quantify the domain of non-interaction between the signal wavelength and the heterogeneity thickness. Only at the shortest periods can the slab crust LVL between upper plate and slab mantle be simply characterized in term of thickness and velocity contrast at its boundaries. For a $7-\mathrm{km}$-thick oceanic crust LVL, only wavelet periods shorter than about $0.8 \mathrm{~s}$ will allow to identify clearly a thin oceanic crust. Going to longer periods will first lead to underestimate the time-thickness of the LVL, then to overestimate it increasingly above twice this period. For a bandpass commonly used in data processing, the LVL will thus appear twice thicker than it is. The amplitudes also vary, increasing from the short period, non-interaction domain where their values only depend on the velocity contrast, to the resonance period, and then decreasing. Inferences on the velocity contrast, as well as using it for the transformation of the time to thickness for this layer will be in error if the domain with respect to resonance is not identified.

The analysis of the response in conversion, then carried out from full waveform synthetic seismograms in a dipping slab model, allows to test and validate a multiresolution approach to real observations. This approach involves building the wavelet response of the slab top as the scalogram of RFs for $P$ wavelets of different scales extracted from observations through application of bandpass filters to the seismograms.

With earthquakes providing wavelet periods shorter than $1 \mathrm{~s}$, the multiscale analysis developed here allows for the first time to constrain the top of the slab at 60-70 km depth under Eastern Peloponnesus to have a crust of 7-8 km thickness, hence the slab segment to be of typical oceanic nature. This is found at $180 \mathrm{~km}$ inboard the subduction backstop. This supports a model of the subduction of purely oceanic lithosphere since 4-5 Myr, overridden at the present fast rate by its backstop of southwestward fastly extending upper plate continental material.

\section{ACKNOWLEDGMENTS}

This research is supported by the European Union FP6 NEST (New and Emerging Science and Technology)-INSIGHT programme, under project 'THALES WAS RIGHT' no. 029080, as well as national funding of participating institutions that provided also their instruments: IPGParis, CSIC Barcelona, National Observatory of Athens, Univ. Trieste, ETH Zürich, as well as that from national pools such 
as CNRS-INSU (pools Refraction, Lithoscope and RLBM). IPG Paris contribution IPGP3064.

\section{REFERENCES}

Berkhout, A.J., 1977. Least-squares inverse filtering and wavelet deconvolution, Geophysics, 42, 1369-1383.

Bostock, M.G., Hyndmann, R.D., Rondenay, S. \& Peacock, S.M., 2002. An inverted continental Moho and serpentinization of the forearc mantle, Nature, 417, 536-538.

Castillo, J., Mocquet, A. \& Saracco, G., 2001. Wavelet transform: a tool for the interpretation of upper mantle converted phases at high frequency, Geophys. Res. Lett., 28, 4327-4330.

Chamot-Rooke, N., Rangin, C., Le Pichon, X. \& Dotmed Working Group, 2005. DOTMED: a synthesis of deep marine data in eastern Mediterranean, Mém. Soc. Géol. France, 177, 64p., 9pl.

Christensen, N.I., 2004. Serpentine, peridotites and seismology, Int. Geol. Rev., 46, 795-816.

Clément Ch., Hirn, A., Charvis, Ph., Sachpazi, M. \& Marnelis, F., 2000. Seismic structure and the active Hellenic subduction in the Ionian Islands, Tectonophysics, 329, 141-156.

de Voogd, N. \& den Roijen, H., 1983. Thin-layer response and spectral bandwidth, Geophysics, 48, 12-18.

de Voogd, B., Truffert, C., Chamot-Rooke, N., Huchon, P., Lallemant, S. \& Le Pichon, X., 1992. Two-ship deep seismic sounding in the basins of the eastern Mediterranean Sea (Pasiphae Cruise), Geophys. J. Int., 109, $536-552$.

Ferris, A., Abers, G.A., Christensen, D.H. \& Veenstra, E., 2003. High resolution image of the subducted Pacific (?) plate beneath central Alaska, 50-150 km depth, Earth planet. Sci. Lett., 214, 575-588.

Fuchs, K., 1969. On the properties of deep crustal reflectors, Z. Geophys., 35, 133-149.

Gesret, A., 2008. Résolution fine du toit de la subduction Héllénique par les ondes télésismiques converties, Thesis, Institut de Physique du Globe de Paris, pp. 146.

Herrmann, F.J., \& Bernabé, Y., 2004. Seismic singularities at upper-mantle phase transitions: a site percolation model, Geophys. J. Int., 159, 949 960.

Herrmann, F.J., Lyons, W.J. \& Stark, C.P., 2001. Seismic facies characterization by monoscale analysis, Geophys. Res. Lett., 28, 3781-3784.

Hirn, A., Steinmetz, L., Kind, R., \& Fuchs, K., 1973. Long-range profiles in Western Europe: II. Fine structure of the lower lithosphere in France (southern Bretagne), Z. Geophys., 39, 363-384.

Hirn, A., Sachpazi, M., Siliqi, R., Mc Bride, J., Marnelis, M. \& the STREAMERS/PROFILES Group, 1996. A traverse of the Ionian Islands front with coincident normal incidence and wide-angle seismics, Tectonophysics, 264, 35-49.

Hollenstein, Ch., Müller, M.D., Geiger, A. \& Kahle, H.-G., 2008. Crustal motion and deformation in Greece from a decade of GPS measurements, 1993-2003, Tectonophysics, 449, 17-40.

Holschneider, M., 1995. Wavelets: An Analysis Tool, Clarendon, Oxford, England, pp. 423.

Jones, K.A., Warner, M., Le Meur, D., Pascal, G., Tay, P.L. \& the IMERSE Working Group, 2002. Wide-angle images of the Mediterranean backstop structure, Mar. Geol., 186, 145-166.

Kahle, H.-G., Cocard, M., Peter, Y., Geiger, A., Reilinger, R., Barka, A. \& Veis, G., 2000. GPS-derived strain rate field within the boundary zones of the Eurasian, African, and Arabian plates, J. geophys. Res., 105, $23353-23370$.

Kallweit, R.S. \& Wood, L.C., 1982. The limits of resolution of zero-phase wavelets, Geophysics, 47, 1035-1046.

Kawakatsu, H. \& Watada, S., 2007 Seismic evidence for deep-water transportation in the mantle, Science, 316, 1468-1471.

Kennet, B.L.N., 1980. Seismic waves in a stratified medium. II. Theoretical seismograms, Geophys. J. R. astr. Soc., 57, 557-583.

Kind, R., Kosarev, G.L. \& Petersen, N.V., 1995. Receiver functions at the stations of the German Regional Seismic network (GRSN), Geophys. J. Int., 121, 191-202.
Laigle, M., Hirn, A., Sachpazi, M. \& Clément, C., 2002. Seismic coupling and structure of the Hellenic subduction in the Ionian islands region, Earth planet. Sci. Lett., 200, 243-253.

Laigle, M., Sachpazi, M. \& Hirn, A., 2004. Variation of seismic coupling with slab detachment and upper plate structure along the Hellenic subduction, Tectonophysics, 391, 97-108.

Le Gonidec, Y. \& Gibert, D., 2007. Multiscale analysis of waves reflected by granular media: acoustic experiments on glass beads and effective medium theories, J. geophys. Res., 112, B05103, doi:10.1029/2006JB004518.

Le Gonidec, Y., Gibert, D., \& Proust, J., 2002. Multiscale analysis of waves reflected by complex interfaces: basic principles and experiments, J. geophys. Res., 107(B9), 2184, doi:10.1029/2001JB000558.

Le Pichon, X., Chamot-Rooke, N., Lallemant, S., Noomen, R. \& Veis, G., 1995. Geodetic determination of the kinematics of central Greece with respect to Europe: implications for eastern Mediterranean tectonics, $J$. geophys. Res., 100, 12 675-12 690.

Li, X., Sobolev, S.V., Kind, R., Yuan, X. \& Estabrook, C., 2000. A detailed receiver function image of the upper mantle discontinuities in the Japan subduction zone, Earth planet. Sci. Lett., 183, 527-541.

Li, X. et al., 2003. Receiver function study of the Hellenic subduction zone: imaging crustal thickness variations and the oceanic Moho of the descending African lithosphere, Geophys. J. Int., 155, 733748.

Lombardi, D., Braunmiller, J., Kissling E. \& Giardini, D., 2008. Moho depth and Poisson's ration in the Western-Central Alps from receiver functions, Geophys. J. Int., 173, 249-264.

Mallat, S. \& Hwang, W.L., 1992. Singularity detection and processing with wavelets, IEEE Trans. Inform. Theory, 38, 617-643.

McClusky, S. et al., 2000. Global Positioning System constraints on plate kinematics and dynamics in the eastern Mediterranean and Caucasus, $J$. geophys. Res., 105, 5695-5719.

Nicholson, T., Bostock, M. \& Cassidy, J.F., 2005. New constraints on subduction structure in northern Cascadia, Geophys. J. Int., 161, 849859.

Nyst, M. \& Thatcher, W., 2004. New constraints on the active tectonic deformation of the Aegean, J. geophys. Res., 109, B11406.

Papanikolaou, D. \& Royden, L.H., 2007. Disruption of the Hellenic arc: late Miocene extensional detachment faults and steep Pliocene-Quaternary normal faults - or what happened at Corinth? Tectonics, 26, TC5003, doi: 10.1029/2006TC002007.

Papazachos, B.C., Karakostas, V.G., Papazachos, C.B. \& Scordilis, E.M., 2000. The geometry of the Wadati-Benioff zone and lithospheric kinematics in the Hellenic arc, Tectonophysics, 319, 275-230.

Polonia, A., Camerlenghi, A., Davey, F. \& Sorti, F., 2002. Accretion, structural style and syn-continental sedimentation in the Eastern Mediterranean Sea, Mar. Geol., 186, 127-144.

Ranero, C., Morgan, J.P., McIntosh, K., \& Reichert, C. 2003. Bendingrelated faulting and mantle serpentinization at the Middle America trench, Nature, 425, 367-373.

Reston, T.J., von Huene, R., Dickmann, T., Klaeschen, D. \& Kopp, H., 2002. Frontal accretion along the western Mediterranean Ridge: the effect of Messinian evaporites on the wedge mechanics and structural style, Mar. Geol., 186, 59-82.

Reynard, B., Hilairet, N., Balan, E. \& Lazzeri, M., 2007. Elasticity of serpentines and extensive serpentinization in subduction zones, Geophys. Res. Lett., 34, L13307, doi:10.1029/2007GL030176.

Rondenay, S., Abers, G.A. \& van Keken, P., 2008. Seismic imaging of subduction zone metamorphism, Geology, 36, 275-278.

Royden, L. H., 1993. Evolution of retreating subduction boundaries formed during continental collision, Tectonics, 12, 629-638.

Royden, L.H. \& Husson, L., 2006. Trench motion, slab geometry and viscous stresses in subduction systems, Geophys. J. Int., 167, 881-905.

Sachpazi, M., et al., 2000. Western Hellenic subduction and Cephalonia transform: local earthquakes and plate transport and strain, Tectonophysics, 319, 301-319.

Sachpazi, M. et al., 2007. Moho topography under central Greece and its compensation by Pn time-terms for the accurate location of hypocenters: 
the example of the Gulf of Corinth 1995 Aigion earthquake, Tectonophysics, 440, 53-65.

Sodoudi, S. et al., 2006. Lithospheric structure of the Aegean obtained from $\mathrm{P}$ and $\mathrm{S}$ receiver functions, J. geophys. Res., 111, B12307, doi: 10.1029/2005JB003932.

Spakman, W., 1986. Subduction beneath Eurasia in connection with the Mesozoic Tethys, Geologie en Mijnbouw, 65, 145-153.

Spakman, W., Van Der Lee, S. \& Van Der Hilst, R., 1993. Travel-time tomography of the European-Mediterranean mantle down to $1400 \mathrm{~km}$, Phys. Earth planet. Int., 79, 3-74.

Stammler, K., 1993. Seismic Handler-Programmable multichannel data handler for interactive and automatic processing of seismological analyses, Comput. Geosci., 19, 135-140.

Suckale, J., Rondenay, S., Sachpazi, M., Charalampakis, M., Hosa, A. \& Royden, L.H., 2009. High-resolution seismic imaging of the western Hellenic subduction zone using teleseismic scattered waves, Geophys. $J$. Int., doi:10.1111/j.1365-246X.2009.04170x.

Taymaz, T., Jackson, J. \& Westaway, R., 1990. Earthquake mechanisms in the Hellenic Trench near Crete, Geophys. J. Int., 102, 695-731.

Truffert, C., Chamot-Rooke, N., Lallemant, S., de Voogd, B., Huchon, P. \& Le Pichon, X., 1993. The crust of the western Mediterranean Ridge from deep seismic data and application to field data from the northern Rio Grande Rift, Geophys. J. Int., 125, 443-458.

Underhill, J., R., 1989. Late Cenozoic deformation of the Hellenides foreland, western Greece, Geol. Soc. Am. Bull., 101, 613-634.

van Hinsbergen, D.J.J., Hafkenscheid, E., Spakman, W., Meulenkamp, J.E. $\&$ Wortel, R., 2005. Nappe stacking resulting from subduction of oceanic and continental lithosphere below Greece, Geology, 33, 325-328.

Virieux, J., 1986. P-SV wave propagation in heterogeneous media: velocitystress finite-difference method, Geophysics, 51, 889-899.

White, R.S., McKenzie, D. \& O'Nions, R.K., 1992. Oceanic crustal thickness from seismic measurements and rare Earth element inversions, $J$. geophys. Res., 97, 19 683-19 715.

Widess, M.B., 1973. How thin is a thin bed?, Geophysics, 38, 1176-1180.

Wortel, M.J.R. \& Spakman, W., 2000. Subduction and slab detachement in the Mediterranean-Carpathian region, Science, 290, 1910-1917.

Wyss, M. \& Baer, M., 1981. Earthquake hazard in the Hellenic arc, in Earthquake Prediction: An international Review, Vol. 4, pp. 153-172, eds Simpson, D.W. \& Richards, P.G., AGU Maurice Ewing Series, Am. geophys. Union, Washington, D.C.

Yuan, X. et al., 2000. Subduction and collision processes in the Central Andes constrained by converted seismic phases, Nature, 408, 958-961. 